SPT-04/086

\title{
Topological expansion for the 1-hermitian matrix model correlation functions.
}

\author{
B. Eynard ${ }^{1}$ \\ Service de Physique Théorique de Saclay, \\ F-91191 Gif-sur-Yvette Cedex, France.
}

\begin{abstract}
:
We rewrite the loop equations of the hermitian matrix model, in a way which involves no derivative with respect to the potential, we compute all the correlation functions, to all orders in the topological $1 / N^{2}$ expansion, as residues on an hyperelliptical curve. Those residues, can be represented as Feynmann graphs of a cubic field theory on the curve.
\end{abstract}

\section{Introduction}

We consider the formal hermitian matrix integral:

$$
Z=\int_{H_{N}} \mathrm{~d} M \mathrm{e}^{-N \operatorname{tr} V(M)}
$$

where $M$ is a $N \times N$ hermitian matrix, $\mathrm{d} M$ is the product of Lebesgue measures of all real components of $M . V(x)$ is a polynomial of degree $d+1 \geq 2$ called the potential. $Z$ is called the partition function.

Our goal is to compute the large $N$ limit, as well as the full $1 / N^{2}$ expansion, of the following formal expectation values $(<>$ is the average computed with the probability measure $\left.\frac{1}{Z} \mathrm{e}^{-N \operatorname{tr} V(M)} d M\right)$ :

$$
\bar{W}_{k}\left(x_{1}, \ldots, x_{k}\right):=N^{k-2}\left\langle\operatorname{tr} \frac{1}{x_{1}-M} \operatorname{tr} \frac{1}{x_{2}-M} \ldots \operatorname{tr} \frac{1}{x_{k}-M}\right\rangle_{\mathrm{c}}
$$

\footnotetext{
${ }^{1}$ E-mail: eynard@spht.saclay.cea.fr
} 


$$
W_{k}\left(x_{1}, \ldots, x_{k}\right):=\lim _{N \rightarrow \infty} \bar{W}_{k}\left(x_{1}, \ldots, x_{k}\right)
$$

where the subscript $c$ means connected part or cumulant.

When $Z$ is considered as a formal generating function, it is well known [1] that the correlation function $\bar{W}_{k}\left(x_{1}, \ldots, x_{k}\right)$ has a $1 / N^{2}$ expansion, also called topological expansion, noted:

$$
\bar{W}_{k}\left(x_{1}, \ldots, x_{k}\right):=\sum_{h=0}^{\infty} N^{-2 h} W_{k}^{(h)}\left(x_{1}, \ldots, x_{k}\right)
$$

Let us emphasize that in general $Z$ is not a convergent integral, the partition function as well as the $\bar{W}_{k}$ 's are to be understood as formal series in the coefficients of the potential, see [1] for details.

In that formal sense, the expectation value of a product of $k$ traces is the combinatoric generating function for enumerating discrete surfaces with $k$ holes, and the variables $x_{1}, \ldots, x_{k}$ are fugacities for the lengths of the $k$ boundaries [23, 9, 8, 24, It is well known [11] that the power of $N$ associated to each discrete surface is its Euler characteristic $\chi$. For a genus $h$ connected surface with $k$ holes, we have $\chi=2-k-2 h$. This is why the quantity 1.2 has a large $N$ limit. The large $N$ limit $1.3 W_{k}\left(x_{1}, \ldots, x_{k}\right)=W_{k}^{(0)}\left(x_{1}, \ldots, x_{k}\right)$ is therefore the generating function of genus zero discrete surfaces with $k$ boundaries, and each $W_{k}^{(h)}\left(x_{1}, \ldots, x_{k}\right)$ is the generating function of genus $h$ discrete surfaces with $k$ boundaries.

The problem of computing the $\bar{W}_{k}$ 's has been addressed many times, for various applications to physics and mathematics. Indeed, the correlation functions of eigenvalues (and thus of traces of powers) of a random matrix have a universal behavior which (this is what universality means) is observed in many physical phenomena, ranging from solid state physics (quantum chaos, mesoscopic conductors, see [30, 25] ) to high energy physics (nuclear physics [30], Quantum chromodynamics 32], string theory [12]), and in mathematics (distribution of Riemann zeta's zeroes [6]).

In the 90's, random matrices were extensively studied in the context of quantum gravity (see [11]), which is nothing but statistical physics on random discretized surfaces, i.e. the combinatorial problem of enumerating discrete surfaces of given topology, as described above.

Quantum gravity is also deeply related to conformal field theory (CFT), when one takes a "double scaling limit" where very large discrete surfaces are dominant, in other words, CFT is the limit of continuous surfaces. Depending on the limit chosen, and on the coefficients of the potential $V(x)$, one may reach different double scaling limits, which are in relationships with the $(p, q=2)$ minimal models in CFT. All the critical exponents of such surfaces are given by KPZ's formula [27]. 
It is thus expected, that in appropriate double scaling limits, expectation values of the form 1.2 can be computed from a quantum field theory, namely Liouville's theory.

Here, without taking any double scaling limit,we will find a quantum-field-theorylike Feynmann expansion for the $\bar{W}_{k}\left(x_{1}, \ldots, x_{k}\right)$.

The $W_{k}$ 's have been computed in the literature by various methods. A formula of Dyson [14 gives the $\bar{W}_{k}$ 's for finite $N$ in terms of orthogonal polynomials, but is not very convenient for large $N$ limit calculations, and is not convenient for the formal model. The method of loop equations [11, 31, 2, 3, 19, 16] gives recursion relations between the $\bar{W}_{k}$ 's, which simplify in the large $N$ limit. The loop equations have been known for a while, and give a very effective algorithm for computing explicitly the $W_{k}$ 's (see [2, 1, 3]). The method developed by [2, 1] for computing the $W_{k}$ 's, consists in computing $W_{1}$ and then obtain the $W_{k}$ 's by taking iterated derivatives with respect to the potentials (loop insertion method). This method has two drawbacks: first in order to find $W_{k+1}$, one should know $W_{k}$ for all potentials (in particular one must take infinite degree potentials); second, before computing $W_{k}$, one has to compute $W_{1}, W_{2}, \ldots, W_{k-1}$, i.e. it has not been found how to integrate the recursion formulae of [1].

Here we consider new loop equations, which allow to find recursion relations between the $W_{k}$ 's, without taking any derivatives with respect to the potential (we may work with fixed potential). Moreover, the recursion relations for the $W_{k}^{(h)}$ 's obtained in this paper can be integrated: the $k^{\text {th }}$-loop function to order $N^{-2 h}$ is a $k$-legs, $h$-loops Feynmann graph of a $\phi^{3}$ theory living on an hyperelliptical curve.

\section{Outline:}

- in section 2 we introduce the notations.

- in section 3 we introduce some basic tools of algebraic geometry.

- in section 4 we write and solve the loop equations to large $N$ leading order, i.e. we compute the $W_{k}$ 's.

- in section 5 we write and solve the loop equations recursively to each order in $1 / N^{2}$, i.e. we compute the $W_{k}^{(h)}$ 's.

- in section 6] we do explicitly the computation in the one-cut case.

- in section [7we conclude by presenting perspectives of applications to other matrix models (2 matrix model).

\section{Definitions and notations}

From now on, we assume that $V^{\prime}(x)$ is monic of degree $d \geq 1$. 


\subsection{Loop functions}

For $k \geq 1$, we define (the subscript $c$ means connected part or cumulant):

$$
\begin{gathered}
\bar{W}_{k}\left(x_{1}, \ldots, x_{k}\right):=N^{k-2}\left\langle\operatorname{tr} \frac{1}{x_{1}-M} \operatorname{tr} \frac{1}{x_{2}-M} \ldots \operatorname{tr} \frac{1}{x_{k}-M}\right\rangle_{\mathrm{c}} \\
\bar{U}_{k}\left(x_{1} ; x_{2}, \ldots, x_{k}\right):=N^{k-2}\left\langle\operatorname{tr} \frac{V_{1}^{\prime}\left(x_{1}\right)-V_{1}^{\prime}\left(M_{1}\right)}{x_{1}-M} \operatorname{tr} \frac{1}{x_{2}-M} \ldots \operatorname{tr} \frac{1}{x_{k}-M}\right\rangle_{\mathrm{c}}
\end{gathered}
$$

and their large $N$ limits:

$$
\begin{gathered}
W_{k}\left(x_{1}, \ldots, x_{k}\right):=W_{k}^{(0)}\left(x_{1}, \ldots, x_{k}\right):=\lim _{N \rightarrow \infty} \bar{W}_{k}\left(x_{1}, \ldots, x_{k}\right) \\
U_{k}\left(x_{1} ; x_{2}, \ldots, x_{k}\right):=U_{k}^{(0)}\left(x_{1} ; x_{2}, \ldots, x_{k}\right):=\lim _{N \rightarrow \infty} \bar{U}_{k}\left(x_{1} ; x_{2}, \ldots, x_{k}\right)
\end{gathered}
$$

as well as their formal $1 / N^{2}$ expansions $(k \geq 1, h \geq 0)$ :

$$
\begin{aligned}
\bar{W}_{k}\left(x_{1}, \ldots, x_{k}\right) & :=\sum_{h=0}^{\infty} N^{-2 h} W_{k}^{(h)}\left(x_{1}, \ldots, x_{k}\right) \\
\bar{U}_{k}\left(x_{1} ; x_{2}, \ldots, x_{k}\right) & :=\sum_{h=0}^{\infty} N^{-2 h} U_{k}^{(h)}\left(x_{1} ; x_{2}, \ldots, x_{k}\right)
\end{aligned}
$$

Notice $U_{1}^{(0)}$ is a monic polynomials of degree $d-1$, and as soon as $k+h \geq 2, U_{k}^{(h)}$ is a polynomial of degree at most $d-2$ in $x_{1}$. We have:

$$
U_{k}^{(h)}\left(x_{1} ; x_{2}, \ldots, x_{k}\right)=\underset{x_{1} \rightarrow \infty}{\operatorname{Pol}} V_{1}^{\prime}\left(x_{1}\right) W_{k}^{(h)}\left(x_{1}, \ldots, x_{k}\right)
$$

The functions $\bar{W}_{k}$ are called loop-functions, because they are generating functions for discrete surfaces with $k$ boundaries, i.e. $k$ loops.

\section{$2.2 \quad$ Filling fractions}

If the integral 1.1 were to be considered as a convergent integral, the $1 / N^{2}$ expansion would exist only in the so-called one-cut case (see [7, 10]). Here 1.1] is considered as a formal power series, by its expansion in the vicinity of a minimum of the potential $\operatorname{tr} V(M)$. The potential $V(x)$ has in general $d=\operatorname{deg} V^{\prime}$ extrema, and thus, the potential $\operatorname{Tr} V(M)$ can have extrema indexed by the number of eigenvalues of $M$ lying in the vicinity of each extrema of $V(x)$. The formal perturbative expansion around such local extrema cannot change the number of eigenvalues near each extrema. The fractions of eigenvalues near each extrema of $V$ are called filling fractions, and are thus moduli characterizing the vacuum near which the perturbative formal expansion is computed. 
The filling fractions play an important role in recent applications of random matrix models to string theory [12].

The filling fractions are denoted:

$$
\epsilon_{1}, \epsilon_{2}, \ldots, \epsilon_{s}, \quad \sum_{j=1}^{s} \epsilon_{j}=1
$$

It is well known [2, 11] (and we recover it below) that the function $W_{1}(x)$ is solution of an algebraic equation, it has $s$ cuts $\left[a_{2 j-1}, a_{2 j}\right], j=1, \ldots, s$, which correspond to the location of eigenvalues in the large $N$ limit. The condition that the filling fractions are given can be written:

$$
\forall j=1, \ldots, s, \quad \frac{1}{2 i \pi} \oint_{\left[a_{2 j-1}, a_{2 j}\right]} W_{1}(x) \mathrm{d} x=\epsilon_{j}
$$

where the contour surrounds the segment $\left[a_{2 j-1}, a_{2 j}\right]$ in the trigonometric direction.

Let us for a moment, use the method of [1, 2] for finding the filling fraction conditions for other loop functions.

If $V(x)=\sum_{k} t_{k} x^{k}$, from [1] we introduce the loop insertion operator ${ }^{2}$ :

$$
\frac{\partial}{\partial V(x)}:=-\sum_{k=1}^{\infty} \frac{1}{x^{k+1}} \frac{\partial}{\partial t_{k}}
$$

we then have 2]:

$$
W_{k+1}\left(x_{1}, \ldots, x_{k}, x_{k+1}\right)=\frac{\partial}{\partial V\left(x_{k+1}\right)} W_{k}\left(x_{1}, \ldots, x_{k}\right)
$$

and thus, since the filling fractions are given parameters independent of $V$ and $N$, we must have for all $k \geq 1, h \geq 0, h+k>1$ :

$$
\forall j=1, \ldots, s, \quad \oint_{\left[a_{2 j-1}, a_{2 j}\right]} W_{k}^{(h)}\left(x_{1}, x_{2}, \ldots, x_{k}\right) \mathrm{d} x_{1}=0
$$

From the same argument, since we assume that there is no eigenvalue elsewhere in the complex plane, we can write, for any $m$ in the complex plane, away from the cuts:

$$
\oint_{m} W_{1}\left(x_{1}\right) \mathrm{d} x_{1}=0
$$

(where the contour integral is a small circle around $m$ ) and thus:

$$
\oint_{m} W_{k}^{(h)}\left(x_{1}, x_{2}, \ldots, x_{k}\right) \mathrm{d} x_{1}=0
$$

\footnotetext{
${ }^{2}$ The loop insertion operator $\sum_{k=1}^{\infty} \frac{1}{x^{k+1}} \frac{\partial}{\partial t_{k}}$ is a formal notation which makes sense order by order in the $1 / x$ expansion, and eq 2.12 is perfectly rigorously proven.
} 


\section{The one-loop function and algebraic geometry}

\subsection{The one-loop function}

It is well known [11, 2] (and it is re-derived below) that the one loop function is algebraic:

$$
\begin{aligned}
W_{1}(x) & =\frac{1}{2}\left(V^{\prime}(x)-M(x) \sqrt{\sigma(x)}\right) \\
U_{1}(x) & =\frac{1}{4}\left(V^{\prime 2}(x)-M^{2}(x) \sigma(x)\right)
\end{aligned}
$$

where $M$ and $\sigma$ are monic polynomials (remember $V^{\prime}(x)$ is monic), determined by:

$$
W_{1}(x) \underset{x \rightarrow \infty}{\sim} \frac{1}{x}
$$

and by 2.9 which can be rewritten as follows: let $a_{1}, \ldots, a_{2 s}$ be the zeroes of $\sigma$ :

$$
\sigma(x)=\prod_{i=1}^{2 s}\left(x-a_{i}\right)
$$

we must have:

$$
\forall j \in[1, s-1], \quad \int_{a_{2 j-1}}^{a_{2 j}} M(x) \sqrt{\sigma(x)} \mathrm{d} x=2 i \pi \epsilon_{j}
$$

For a given $s \in[1, d]$, the equations 3.3 and 3.5 give a finite number of solutions for $M$ and $\sigma$. Let us assume that we have chosen one of them.

\subsection{More notations}

For convenience we introduce $m_{1}, \ldots, m_{d-s}$ the zeroes of $M$ :

$$
M(x)=\prod_{i=1}^{d-s}\left(x-m_{i}\right)
$$

We also define for $k \geq 1, h \geq 0$, and $h+k>1$ :

$$
F_{k}^{(h)}\left(x_{1}, \ldots, x_{k}\right):=\left(2^{k} W_{k}\left(x_{1}, \ldots, x_{k}\right)+\frac{2 \delta_{k, 2} \delta_{h, 0}}{\left(x_{1}-x_{2}\right)^{2}}\right) \prod_{i=1}^{k} \sqrt{\sigma\left(x_{i}\right)}
$$

and:

$$
F_{k}\left(x_{1}, \ldots, x_{k}\right):=F_{k}^{(0)}\left(x_{1}, \ldots, x_{k}\right)
$$

It is well known that the $F_{k}$ 's and $F_{k}^{(h)}$ 's are rational functions of all their arguments (see [1, 2, [3]). 
Another useful notation is in terms of multi-linear differential forms:

$$
G_{k}\left(x_{1}, \ldots, x_{k}\right):=W_{k}\left(x_{1}, \ldots, x_{k}\right) d x_{1} \ldots d x_{k}
$$

and for higher orders:

$$
G_{k}^{(h)}\left(x_{1}, \ldots, x_{k}\right):=W_{k}^{(h)}\left(x_{1}, \ldots, x_{k}\right) d x_{1} \ldots d x_{k}
$$

It is well known that they are all multi-linear differentials defined on an hyperelliptical surface. All of them, except $G_{1}$ and $G_{2}$, have poles only at the branch-points (i.e. the zeroes of $\sigma$ ), and have vanishing contour integrals around the cuts.

All this is re-derived below.

\subsection{Hyperelliptical surfaces}

We need to introduce some basic notions of algebraic geometry [21, 22].

Equation 3.1 defines an hyperelliptical surface of genus $s-1$. Let $y=V^{\prime}(x)-$ $2 W_{1}(x)$, we have:

$$
y^{2}=M^{2}(x) \sigma(x)
$$

That equation defines a Riemann surface with two sheets (corresponding to the two determinations of the square root). In other word, for each $x$, there are two values of $y(x)$.

Let us define the physical sheet as the sheet where:

$$
x^{-s} \sqrt{\sigma(x)} \underset{x \rightarrow \infty}{\sim}+1
$$

and the second sheet as the one where:

$$
x^{-s} \sqrt{\sigma(x)} \underset{x \rightarrow \infty}{\sim}-1
$$

If $x$ is a point in the physical sheet, let us note $\bar{x}$ the point corresponding to the same $x$ in the second sheet. By definition, we have:

$$
\sqrt{\sigma(\bar{x})}=-\sqrt{\sigma(x)} \quad, \quad M(\bar{x})=M(x) \quad, \quad y(\bar{x})=-y(x) \quad, \quad d \bar{x}=d x
$$

The branch points $a_{i}$ are the points where the two sheets meet, they are such that:

$$
\forall i=1, \ldots, 2 s, \quad \bar{a}_{i}=a_{i}
$$

Near a branch point $a_{i}$, the surface is better parameterized by the local coordinate:

$$
\tau_{i}(x):=\sqrt{x-a_{i}}=-\tau_{i}(\bar{x})
$$


i.e.

$$
x=a_{i}+\tau_{i}^{2} \quad, \quad d x=2 \tau_{i} d \tau_{i}
$$

In particular, the differential $d x$ has a (simple) zero at $x=a_{i}$.

Holomorphic differentials: Let $L(x)$ be any polynomial of degree $\leq s-2$. Since $\sqrt{\sigma(x)}$ has a simple zero at $x=a_{i}$, the differential $L(x) \frac{d x}{\sqrt{\sigma(x)}}$ has no singularity on the whole surface (neither near the branch points, nor at $\infty$ ), it is thus called a holomorphic differential. One has the following classical theorem: there exist a unique set of $s-1$ polynomials of degree $s-2$, which we note $L_{j}(x)$, such that:

$$
\forall l, j \in[1, s-1]^{2}, \quad \oint_{\left[a_{2 l-1}, a_{2 l}\right]} \frac{L_{j}(x)}{\sqrt{\sigma(x)}} \mathrm{d} x=2 i \pi \delta_{l, j}
$$

The differentials $\frac{L_{j}(x)}{\sqrt{\sigma(x)}} d x$ are called the normalized holomorphic differentials. Notice that the $L_{j}$ 's form a basis of degree $\leq s-2$ polynomials. For any polynomial $P(x)$ such that $\operatorname{deg} P \leq s-2$, we have:

$$
P(x)=\sum_{j=1}^{s-1}\left(\frac{1}{2 i \pi} \oint_{\left[a_{2 j-1}, a_{2 j}\right]} \frac{P\left(x^{\prime}\right)}{\sqrt{\sigma\left(x^{\prime}\right)}} \mathrm{d} x^{\prime}\right) L_{j}(x)
$$

Notice that on the $s^{\text {th }}$ cut, we have:

$$
\forall j \in[1, s-1], \quad \oint_{\left[a_{2 s-1}, a_{2 s}\right]} \frac{L_{j}(x)}{\sqrt{\sigma(x)}} \mathrm{d} x=-2 i \pi
$$

We define:

$$
L_{s}(x):=0
$$

so that 3.19 holds also with the sum on $j$ running from 1 to $s$.

Normalized differential of the third kind: For any $x^{\prime}$ on the curve, there exists a unique meromorphic differential, noted $d S\left(x, x^{\prime}\right)$, which has only two simple poles in $x$, located at $x=x^{\prime}$ and $x=\overline{x^{\prime}}$, and such that:

$$
\left\{\begin{array}{l}
d S\left(x, x^{\prime}\right) \underset{x \rightarrow x^{\prime}}{\sim} \frac{d x}{x-x^{\prime}}+\text { finite } \\
d S\left(x, x^{\prime}\right) \underset{x \rightarrow \bar{x}^{\prime}}{\sim}-\frac{d x}{x-x^{\prime}}+\text { finite } \\
\forall j=1, \ldots, s-1, \quad \oint_{\left[a_{2 j-1}, a_{2 j}\right]} d S\left(x, x^{\prime}\right)=0
\end{array}\right.
$$

Notice that $d S\left(x, x^{\prime}\right)$ is a meromorphic differential in the variable $x$, and a multi-valued function of the variable $x^{\prime}$. 
It is easy to check that we have the following expression:

$$
d S\left(x, x^{\prime}\right)=\frac{\sqrt{\sigma\left(x^{\prime}\right)}}{\sqrt{\sigma(x)}}\left(\frac{1}{x-x^{\prime}}-\sum_{j=1}^{s-1} C_{j}\left(x^{\prime}\right) L_{j}(x)\right) d x
$$

where

$$
C_{j}\left(x^{\prime}\right):=\frac{1}{2 i \pi} \oint_{\left[a_{2 j-1}, a_{2 j}\right]} \frac{\mathrm{d} x}{\sqrt{\sigma(x)}} \frac{1}{x-x^{\prime}}
$$

In this formula, it is assumed that $x^{\prime}$ lies outside the contours $\left[a_{2 j-1}, a_{2 j}\right]$. One has to be careful when $x^{\prime}$ approaches some branch point $a_{j}$. When $x^{\prime}$ lies inside the contour around $\left[a_{2 j-1}, a_{2 j}\right]$, then one has:

$$
C_{l}\left(x^{\prime}\right)+\frac{\delta_{l, j}}{\sqrt{\sigma\left(x^{\prime}\right)}}=\frac{1}{2 i \pi} \oint_{\left[a_{2 l-1}, a_{2 l}\right]} \frac{\mathrm{d} x}{\sqrt{\sigma(x)}} \frac{1}{x-x^{\prime}}
$$

which is analytical in $x^{\prime}$ when $x^{\prime}$ approaches $a_{2 j-1}$ or $a_{2 j}$.

For $i=1, \ldots, s$, we define:

$$
\begin{aligned}
d S_{2 i-1}\left(x, x^{\prime}\right) & :=d S_{2 i}\left(x, x^{\prime}\right):=d S\left(x, x^{\prime}\right)-\frac{L_{i}(x)}{\sqrt{\sigma(x)}} \\
& =\frac{\sqrt{\sigma\left(x^{\prime}\right)}}{\sqrt{\sigma(x)}}\left(\frac{1}{x-x^{\prime}}-\frac{L_{i}(x)}{\sqrt{\sigma\left(x^{\prime}\right)}}-\sum_{j=1}^{s-1} C_{j}\left(x^{\prime}\right) L_{j}(x)\right) d x
\end{aligned}
$$

which is a one-form in $x$, with poles at $x=x^{\prime}$ and $x=\overline{x^{\prime}}$, and which is analytical in $x^{\prime}$ when $x^{\prime}$ is close to $a_{2 i-1}$ or $a_{2 i}$.

Bergmann kernel: For any $x^{\prime}$ on the curve, there exists a unique bilinear differential, noted $B\left(x, x^{\prime}\right)$, called the Bergmann kernel, which has only one double pole in $x$, located at $x=x^{\prime}$ (in particular no pole at $x=\overline{x^{\prime}}$ ), with no residue, and such that:

$$
\left\{\begin{array}{l}
B\left(x, x^{\prime}\right) \underset{x \rightarrow x^{\prime}}{\sim} \frac{d x d x^{\prime}}{\left(x-x^{\prime}\right)^{2}}+\text { finite } \\
\forall j=1, \ldots, s-1, \quad \oint_{x \in\left[a_{2 j-1}, a_{2 j}\right]} B\left(x, x^{\prime}\right)=0
\end{array}\right.
$$

It is easy to check that $B\left(x, x^{\prime}\right)=B\left(x^{\prime}, x\right)$ and:

$$
\begin{aligned}
B\left(x, x^{\prime}\right) & =\frac{1}{2 \sqrt{\sigma(x)}} d x d x^{\prime} \frac{\partial}{\partial x^{\prime}}\left(\frac{\sqrt{\sigma(x)}+\sqrt{\sigma\left(x^{\prime}\right)}}{x-x^{\prime}}-\sum_{j=1}^{s-1} C_{j}\left(x^{\prime}\right) L_{j}(x) \sqrt{\sigma\left(x^{\prime}\right)}\right) \\
& =\frac{1}{2} d x^{\prime} \frac{\partial}{\partial x^{\prime}}\left(\frac{d x}{x-x^{\prime}}+d S\left(x, x^{\prime}\right)\right)
\end{aligned}
$$

It can be written:

$$
B\left(x, x^{\prime}\right)=\frac{d x d x^{\prime}}{2\left(x-x^{\prime}\right)^{2}}+\frac{Q\left(x, x^{\prime}\right) d x d x^{\prime}}{4\left(x-x^{\prime}\right)^{2} \sqrt{\sigma(x)} \sqrt{\sigma\left(x^{\prime}\right)}}
$$


where $Q\left(x, x^{\prime}\right)$ is a symmetric polynomial in $x$ and $x^{\prime}$, of degree at most $s$, such that $Q(x, x)=2 \sigma(x)$ and $\left.\partial_{x^{\prime}} Q\left(x, x^{\prime}\right)\right|_{x^{\prime}=x}=\sigma^{\prime}(x)$ :

$$
Q\left(x, x^{\prime}\right)=2 \sigma(x)+\left(x^{\prime}-x\right) \sigma^{\prime}(x)+\frac{4}{3} \frac{\left(x^{\prime}-x\right)^{2}}{2} S(x)+O\left(x^{\prime}-x\right)^{3}
$$

where $S(x)$ is called projective connection at $x$. We can write:

$$
Q\left(x, x^{\prime}\right)=2 \sigma(x)+\left(x^{\prime}-x\right) \sigma^{\prime}(x)+\left(x-x^{\prime}\right)^{2} A\left(x, x^{\prime}\right)
$$

where $A\left(x, x^{\prime}\right)$ is a polynomial in both variables. We have:

$$
\begin{aligned}
\frac{B\left(x, x^{\prime}\right)}{d x d x^{\prime}}= & \frac{1}{2\left(x-x^{\prime}\right)^{2}}+\frac{\sigma(x)}{2\left(x-x^{\prime}\right)^{2} \sqrt{\sigma(x)} \sqrt{\sigma\left(x^{\prime}\right)}} \\
& +\frac{\sigma^{\prime}(x)}{4\left(x^{\prime}-x\right) \sqrt{\sigma(x)} \sqrt{\sigma\left(x^{\prime}\right)}}+\frac{A\left(x, x^{\prime}\right)}{4 \sqrt{\sigma(x)} \sqrt{\sigma\left(x^{\prime}\right)}}
\end{aligned}
$$

\section{Loop equations}

Now, we will introduce a method for computing the $W_{k}^{(h)}$ 's. It is based on the so-called loop equations or Schwinger-Dyson equations, i.e. invariance of the integral 1.1 under local infinitesimal change of variable.

\subsection{Useful notations}

Let $K=\{2, \ldots, k\}$. For any $j \leq k-1$ we denote:

$$
K_{j}:=\{I \subset K / \# I=j\}
$$

and for any subset $I \in K_{j}$ we define:

$$
I=\left\{i_{1}, i_{2}, \ldots, i_{j}\right\} \quad \longrightarrow \quad x_{I}:=x_{i_{1}}, x_{i_{2}}, \ldots, x_{i_{j}}
$$

as well as:

$$
\sqrt{\sigma\left(x_{I}\right)}:=\prod_{l=1}^{j} \sqrt{\sigma\left(x_{i_{j}}\right)}
$$

and

$$
d x_{I}:=\prod_{l=1}^{j} d x_{i_{j}}
$$




\subsection{Loop equations}

The invariance of the matrix integral 1.1 under the change of variable $M \rightarrow M+\eta \delta M$ (see [11, 18, 19] for detailed derivations):

$$
\delta M=\frac{1}{x_{1}-M} \prod_{j=2}^{k} \operatorname{tr} \frac{1}{x_{j}-M}
$$

implies, to first order in $\eta$ :

$$
\begin{array}{ll}
k=1: \quad & \bar{W}_{1}\left(x_{1}\right)^{2}+\frac{1}{N^{2}} \bar{W}_{2}\left(x_{1}, x_{1}\right)=V^{\prime}\left(x_{1}\right) \bar{W}_{1}\left(x_{1}\right)-\bar{U}_{1}\left(x_{1}\right) \\
k \geq 2: \quad & 2 \bar{W}_{1}\left(x_{1}\right) \bar{W}_{k}\left(x_{1}, \ldots, x_{k}\right)+\frac{1}{N^{2}} \bar{W}_{k+1}\left(x_{1}, x_{1}, x_{2}, \ldots, x_{k}\right) \\
& +\sum_{j=1}^{k-2} \sum_{I \in K_{j}} \bar{W}_{j+1}\left(x_{1}, x_{I}\right) \bar{W}_{k-j}\left(x_{1}, x_{K-I}\right) \\
& +\sum_{j=2}^{k} \frac{\partial}{\partial x_{j}} \frac{\bar{W}_{k-1}\left(x_{2}, \ldots, x_{j}, \ldots, x_{k}\right)-\bar{W}_{k-1}\left(x_{2}, \ldots, x_{1}, \ldots, x_{k}\right)}{x_{j}-x_{1}} \\
& =V^{\prime}\left(x_{1}\right) \bar{W}_{k}\left(x_{1}, \ldots, x_{k}\right)-\bar{U}_{k}\left(x_{1} ; x_{2}, \ldots, x_{k}\right)
\end{array}
$$

i.e., to leading order in $1 / N^{2}$ we have:

$$
\begin{array}{ll}
k=1: \quad & W_{1}\left(x_{1}\right)^{2}=V^{\prime}\left(x_{1}\right) W_{1}\left(x_{1}\right)-U_{1}\left(x_{1}\right) \\
k \geq 2: \quad & 2 W_{1}\left(x_{1}\right) W_{k}\left(x_{1}, \ldots, x_{k}\right) \\
& +\sum_{j=1}^{k-2} \sum_{I \in K_{j}} W_{j+1}\left(x_{1}, x_{I}\right) W_{k-j}\left(x_{1}, x_{K-I}\right) \\
& +\sum_{j=2}^{k} \frac{\partial}{\partial x_{j}} \frac{W_{k-1}\left(x_{2}, \ldots, x_{j}, \ldots, x_{k}\right)-W_{k-1}\left(x_{2}, \ldots, x_{1}, \ldots, x_{k}\right)}{x_{j}-x_{1}} \\
& =V^{\prime}\left(x_{1}\right) W_{k}\left(x_{1}, \ldots, x_{k}\right)-U_{k}\left(x_{1} ; x_{2}, \ldots, x_{k}\right)
\end{array}
$$

Notice that it implies 3.1 for $k=1$.

Now assume $k \geq 2$, and using 3.1, we rewrite:

$$
\begin{aligned}
& M\left(x_{1}\right) \sqrt{\sigma\left(x_{1}\right)} W_{k}\left(x_{1}, \ldots, x_{k}\right) \\
& =\sum_{j=1}^{k-2} \sum_{I \in K_{j}} W_{j+1}\left(x_{1}, x_{I}\right) W_{k-j}\left(x_{1}, x_{K-I}\right) \\
& +\sum_{j=2}^{k} \frac{\partial}{\partial x_{j}} \frac{W_{k-1}\left(x_{2}, \ldots, x_{j}, \ldots, x_{k}\right)-W_{k-1}\left(x_{2}, \ldots, x_{1}, \ldots, x_{k}\right)}{x_{j}-x_{1}} \\
& +U_{k}\left(x_{1} ; x_{2}, \ldots, x_{k}\right)
\end{aligned}
$$




\subsection{Case $\mathrm{k}=2$}

For $k=2$, 4.8 reads:

$$
\begin{aligned}
M\left(x_{1}\right) \sqrt{\sigma\left(x_{1}\right)} W_{2}\left(x_{1}, x_{2}\right)= & \frac{\partial}{\partial x_{2}}\left(\frac{W_{1}\left(x_{2}\right)-W_{1}\left(x_{1}\right)}{x_{2}-x_{1}}\right)+U_{2}\left(x_{1} ; x_{2}\right) \\
= & -\frac{1}{2} \frac{\partial}{\partial x_{2}}\left(\frac{\left.M\left(x_{2}\right) \sqrt{\sigma\left(x_{2}\right)}-M\left(x_{1}\right) \sqrt{\sigma\left(x_{1}\right)}\right)}{x_{2}-x_{1}}\right) \\
& +\frac{1}{2} \frac{\partial}{\partial x_{2}}\left(\frac{V^{\prime}\left(x_{2}\right)-V^{\prime}\left(x_{1}\right)}{x_{2}-x_{1}}\right)+U_{2}\left(x_{1} ; x_{2}\right) \\
= & -\frac{1}{2} \frac{\partial}{\partial x_{2}}\left(M\left(x_{1}\right) \frac{\sqrt{\sigma\left(x_{2}\right)}-\sqrt{\sigma\left(x_{1}\right)}}{x_{2}-x_{1}}\right) \\
& -\frac{1}{2} \frac{\partial}{\partial x_{2}}\left(\sqrt{\sigma\left(x_{2}\right)} \frac{M\left(x_{2}\right)-M\left(x_{1}\right)}{x_{2}-x_{1}}\right) \\
& +\frac{1}{2} \frac{\partial}{\partial x_{2}}\left(\frac{V^{\prime}\left(x_{2}\right)-V^{\prime}\left(x_{1}\right)}{x_{2}-x_{1}}\right)+U_{2}\left(x_{1} ; x_{2}\right)
\end{aligned}
$$

which can be written:

$$
\sqrt{\sigma\left(x_{1}\right)} W_{2}\left(x_{1}, x_{2}\right)=-\frac{1}{2} \frac{\partial}{\partial x_{2}}\left(\frac{\sqrt{\sigma\left(x_{2}\right)}-\sqrt{\sigma\left(x_{1}\right)}}{x_{2}-x_{1}}\right)+\frac{R_{2}\left(x_{1} ; x_{2}\right)}{M\left(x_{1}\right)}
$$

where $R_{2}\left(x_{1} ; x_{2}\right)$ is a polynomial in $x_{1}$ of degree at most $d-2$. From 2.14 , we know that the LHS has no pole at the zeroes of $M$, thus

$$
R_{2}\left(x_{1} ; x_{2}\right)=M\left(x_{1}\right) P_{2}\left(x_{1} ; x_{2}\right)
$$

where $P_{2}\left(x_{1} ; x_{2}\right)$ is a polynomial in $x_{1}$, of degree $s-2$. We have:

$$
\sqrt{\sigma\left(x_{1}\right)} W_{2}\left(x_{1}, x_{2}\right)=-\frac{1}{2} \frac{\partial}{\partial x_{2}}\left(\frac{\sqrt{\sigma\left(x_{2}\right)}-\sqrt{\sigma\left(x_{1}\right)}}{x_{2}-x_{1}}\right)+P_{2}\left(x_{1} ; x_{2}\right)
$$

In terms of the function $F_{2}$ introduced in 3.8 we have:

$$
\frac{F_{2}\left(x_{1}, x_{2}\right)}{4 \sqrt{\sigma\left(x_{1}\right)} \sqrt{\sigma\left(x_{2}\right)}}=\frac{1}{2 \sqrt{\sigma\left(x_{1}\right)}} \frac{\partial}{\partial x_{2}} \frac{\sqrt{\sigma\left(x_{2}\right)}}{\left(x_{1}-x_{2}\right)}+\frac{P_{2}\left(x_{1} ; x_{2}\right)}{\sqrt{\sigma\left(x_{1}\right)}}
$$

which proves that $F_{2}$ is a rational function of $x_{1}$, and by symmetry, it is also a rational function of $x_{2}$.

Then using 2.12 as well as 3.19 and 3.24 , we find $P_{2}$ :

$$
\frac{F_{2}\left(x_{1}, x_{2}\right)}{4 \sqrt{\sigma\left(x_{1}\right)} \sqrt{\sigma\left(x_{2}\right)}}=\frac{1}{2} \frac{\partial}{\partial x_{2}} \frac{\sqrt{\sigma\left(x_{2}\right)}}{\sqrt{\sigma\left(x_{1}\right)}}\left(\frac{1}{\left(x_{1}-x_{2}\right)}-\sum_{l} C_{l}\left(x_{2}\right) L_{l}\left(x_{1}\right)\right)
$$


then using 3.23 ,

$$
\frac{F_{2}\left(x_{1}, x_{2}\right)}{4 \sqrt{\sigma\left(x_{1}\right)} \sqrt{\sigma\left(x_{2}\right)}}=\frac{1}{2} \frac{\partial}{\partial x_{2}} \frac{d S\left(x_{1}, x_{2}\right)}{d x_{1}}=\frac{B\left(x_{1}, x_{2}\right)}{d x_{1} d x_{2}}-\frac{1}{2} \frac{1}{\left(x_{1}-x_{2}\right)^{2}}
$$

where we recognize $B$ the Bergmann kernel introduced in 3.27. Finally, we have the two-loop function in the form:

$$
W_{2}\left(x_{1}, x_{2}\right)=\frac{B\left(x_{1}, x_{2}\right)}{d x_{1} d x_{2}}-\frac{1}{\left(x_{1}-x_{2}\right)^{2}}=-\frac{B\left(x_{1}, \overline{x_{2}}\right)}{d x_{1} d x_{2}}
$$

or, using 3.29

$$
F_{2}\left(x_{1}, x_{2}\right)=\frac{Q\left(x_{1}, x_{2}\right)}{\left(x_{1}-x_{2}\right)^{2}}
$$

The result 4.16 or 4.13 is well known and can be found in many places in the literature 15]. We have just presented one derivation for completeness. Now, let us move to higher loop functions.

Remark: we can write

$$
F_{2}\left(x, x_{1}\right)=2 \frac{\sigma(x)}{\left(x-x_{1}\right)^{2}}-\frac{\sigma^{\prime}(x)}{x-x_{1}}+A\left(x, x_{1}\right)
$$

where $A\left(x, x_{1}\right)$ is a polynomial in both variables. It implies:

$$
W_{2}(x, x)=-\frac{\sigma^{\prime \prime}(x)}{8 \sigma(x)}+\frac{\sigma^{\prime}(x)^{2}}{16 \sigma(x)^{2}}+\frac{A(x, x)}{4 \sigma(x)}
$$

which is a rational function of $x$, with double poles at the branch-points.

\section{$4.4 \mathrm{k}=3$}

Starting from 4.8 for $k=3$, i.e.

$$
\begin{aligned}
M\left(x_{1}\right) \sqrt{\sigma\left(x_{1}\right)} W_{3}\left(x_{1}, x_{2}, x_{3}\right)= & 2 W_{2}\left(x_{1}, x_{2}\right) W_{2}\left(x_{1}, x_{3}\right) \\
& +\frac{\partial}{\partial x_{2}} \frac{W_{2}\left(x_{2}, x_{3}\right)-W_{2}\left(x_{1}, x_{3}\right)}{x_{2}-x_{1}} \\
& +\frac{\partial}{\partial x_{3}} \frac{W_{2}\left(x_{2}, x_{3}\right)-W_{2}\left(x_{1}, x_{2}\right)}{x_{3}-x_{1}} \\
& +U_{3}\left(x_{1} ; x_{2}, x_{3}\right)
\end{aligned}
$$

and using the results for $k=2$, we get:

$$
\frac{F_{3}\left(x_{1}, x_{2}, x_{3}\right)}{\sqrt{\sigma\left(x_{2}\right)} \sqrt{\sigma\left(x_{3}\right)}}=\frac{F_{2}\left(x_{1}, x_{2}\right) F_{2}\left(x_{1}, x_{3}\right)}{\sigma\left(x_{1}\right) M\left(x_{1}\right) \sqrt{\sigma\left(x_{2}\right)} \sqrt{\sigma\left(x_{3}\right)}}
$$




$$
\begin{aligned}
& +\frac{8}{M\left(x_{1}\right)} \frac{\partial}{\partial x_{2}} \frac{W_{2}\left(x_{2}, x_{3}\right)}{x_{2}-x_{1}}+\frac{8}{M\left(x_{1}\right)} \frac{\partial}{\partial x_{3}} \frac{W_{2}\left(x_{2}, x_{3}\right)}{x_{3}-x_{1}} \\
& +\frac{8 U_{3}\left(x_{1} ; x_{2}, x_{3}\right)}{M\left(x_{1}\right)}
\end{aligned}
$$

i.e. $F_{3}\left(x_{1}, x_{2}, x_{3}\right)$ is a rational function of $x_{1}$, and by symmetry, it is a rational function of all its arguments. Expression 4.20 clearly shows that $F_{3}$ has no pole when $x_{1}=x_{2}$ or $x_{1}=x_{3}$. Moreover, from 2.14, we know that it has no pole at the zeroes of $M$. Thus the only possible poles of $F_{3}$ are at the branch points and at $\infty$. Notice that only the first term in the RHS of 4.21 has poles at the branch points.

Before continuing, let us study the case $k>3$.

\section{5 $\mathrm{k}$ larger or equal to 3}

Now assume $k>3$. We start from 4.8 .

$$
\begin{aligned}
\sqrt{\sigma\left(x_{1}\right)} W_{k}\left(x_{1}, x_{K}\right)= & \sum_{j=1}^{k-2} \sum_{I \in K_{j}} \frac{W_{j+1}\left(x_{1}, x_{I}\right) W_{k-j}\left(x_{1}, x_{K-I}\right)}{M\left(x_{1}\right)} \\
& +\sum_{i=2}^{k} \frac{\partial}{\partial x_{i}} \frac{W_{k-1}\left(x_{K}\right)-W_{k-1}\left(x_{1}, x_{K-\{i\}}\right)}{\left(x_{i}-x_{1}\right) M\left(x_{1}\right)} \\
& +\frac{U_{k}\left(x_{1} ; x_{K}\right)}{M\left(x_{1}\right)}
\end{aligned}
$$

and we consider separately the terms corresponding to $j=1$ and $j=k-2$ in the RHS, and we write $\sum_{I \in K_{1}}=\sum_{i=2}^{k}$, we get:

$$
\begin{aligned}
\sqrt{\sigma\left(x_{1}\right)} W_{k}\left(x_{1}, x_{K}\right)= & \sum_{j=2}^{k-3} \sum_{I \in K_{j}} \frac{W_{j+1}\left(x_{1}, x_{I}\right) W_{k-j}\left(x_{1}, x_{K-I}\right)}{M\left(x_{1}\right)} \\
& +2 \sum_{i=2}^{k} \frac{\left(W_{2}\left(x_{1}, x_{i}\right)+\frac{1}{2\left(x_{1}-x_{i}\right)^{2}}\right) W_{k-1}\left(x_{1}, x_{K-I}\right)}{M\left(x_{1}\right)} \\
& +\sum_{i=2}^{k} \frac{\partial}{\partial x_{i}} \frac{W_{k-1}\left(x_{K}\right)}{\left(x_{i}-x_{1}\right) M\left(x_{1}\right)}+\frac{U_{k}\left(x_{1} ; x_{K}\right)}{M\left(x_{1}\right)}
\end{aligned}
$$

This clearly proves, by induction on $k$, that for all $k \geq 3, F_{k}\left(x_{1}, x_{K}\right)$ is a rational function of $x_{1}$ (and by symmetry, of all its arguments), with poles only at the branch points and at $\infty$. We have just re-derived it in a way different from [1, 2].

Now, assume $k \geq 3$. Consider the Euclidean division of the polynomial $U_{k}\left(x_{1} ; x_{2}, \ldots, x_{k}\right)$ by $M\left(x_{1}\right)$ :

$$
U_{k}\left(x_{1} ; x_{K}\right)=\frac{2^{-k}}{\sqrt{\sigma\left(x_{K}\right)}} P_{k}\left(x_{1} ; x_{K}\right) M\left(x_{1}\right)+Q_{k}\left(x_{1} ; x_{K}\right)
$$


where $\operatorname{deg} P_{k}=s-2$ and $\operatorname{deg} Q_{k}<d-s$.

Thus, we have found that for any $k \geq 3$, we have:

$$
\frac{F_{k}\left(x_{1}, x_{K}\right)-P_{k}\left(x_{1} ; x_{K}\right)}{\sqrt{\sigma\left(x_{K}\right)}}=\frac{1}{2} \sum_{j=1}^{k-2} \sum_{I \in K_{j}} \frac{F_{j+1}\left(x_{1}, x_{I}\right) F_{k-j}\left(x_{1}, x_{K-I}\right)}{\sigma\left(x_{1}\right) M\left(x_{1}\right) \sqrt{\sigma\left(x_{K}\right)}}+R_{k}\left(x_{1} ; x_{K}\right)
$$

where $R_{k}\left(x_{1} ; x_{K}\right)$ is a rational fraction of $x_{1}$ with no poles at the branch points neither at $\infty$ (it has poles at the zeroes of $M$ and at the $x_{i}$ 's).

\subsection{Cauchy formula}

Cauchy formula gives:

$$
\frac{F_{k}\left(x_{1}, x_{K}\right)-P_{k}\left(x_{1} ; x_{K}\right)}{\sqrt{\sigma\left(x_{K}\right)}}=\operatorname{Res}_{x \rightarrow x_{1}} \frac{d x}{x-x_{1}} \frac{F_{k}\left(x, x_{K}\right)-P_{k}\left(x ; x_{K}\right)}{\sqrt{\sigma\left(x_{K}\right)}}
$$

where the integrand has poles only at the branch points. Therefore we may deform the integration contour used to compute the residue, into residues at the branch points only:

$$
F_{k}\left(x_{1}, x_{K}\right)-P_{k}\left(x_{1} ; x_{K}\right)=\sum_{l=1}^{2 s} \operatorname{Res}_{x \rightarrow a_{l}} \frac{d x}{x_{1}-x}\left(F_{k}\left(x, x_{K}\right)-P_{k}\left(x ; x_{K}\right)\right)
$$

using 4.25 we get the recursion formula for the $F_{k}$ 's, for all $k \geq 3$ :

$$
F_{k}\left(x_{1}, x_{K}\right)-P_{k}\left(x_{1} ; x_{K}\right)=\frac{1}{2} \sum_{l=1}^{2 s} \operatorname{Res}_{x \rightarrow a_{l}} \sum_{j=1}^{k-2} \sum_{I \in K_{j}} \frac{F_{j+1}\left(x, x_{I}\right) F_{k-j}\left(x, x_{K-I}\right)}{\sigma(x) M(x)\left(x_{1}-x\right)} d x
$$

$P_{k}\left(x_{1}, x_{K}\right)$ which is a polynomial in $x_{1}$ of degree at most $s-2$, is computed with formula 3.19 ,

Starting from 2.12, i.e.:

$$
\oint_{\left[a_{2 l-1}, a_{2 l}\right]} \frac{F_{k}\left(x_{1}, x_{K}\right)}{\sqrt{\sigma\left(x_{1}\right)}} d x_{1}=0
$$

we have:

$$
\begin{aligned}
& -\oint_{\left[a_{2 l-1}, a_{2 l}\right]} \frac{P_{k}\left(x_{1} ; x_{K}\right) d x_{1}}{\sqrt{\sigma\left(x_{1}\right)}} \\
= & \sum_{i=1}^{2 s} \sum_{j=1}^{k-2} \sum_{I \in K_{j}} \oint_{\left[a_{2 l-1}, a_{2 l}\right]} \frac{d x_{1}}{\sqrt{\sigma\left(x_{1}\right)}} \operatorname{Res}_{x \rightarrow a_{i}} \frac{F_{j+1}\left(x, x_{I}\right) F_{k-j}\left(x, x_{K-I}\right)}{2\left(x_{1}-x\right) M(x) \sigma(x)} d x \\
= & \frac{1}{2 i \pi} \sum_{i=1}^{s} \sum_{j=1}^{k-2} \sum_{I \in K_{j}} \oint_{\left[a_{2 l-1}, a_{2 l}\right]} \frac{d x_{1}}{\sqrt{\sigma\left(x_{1}\right)}} \oint_{\left[a_{2 i-1}, a_{2 i}\right]} \frac{F_{j+1}\left(x, x_{I}\right) F_{k-j}\left(x, x_{K-I}\right)}{2\left(x_{1}-x\right) M(x) \sigma(x)} d x
\end{aligned}
$$


Notice that for $i=l$, the contour of integration of $x_{1}$ encloses the contour of $x$. We may exchange the position of the two contours, by picking a residue at $x_{1}=x$, thus:

$$
\begin{aligned}
& -\oint_{\left[a_{2 l-1}, a_{2 l}\right]} \frac{P_{k}\left(x_{1} ; x_{K}\right) d x_{1}}{\sqrt{\sigma\left(x_{1}\right)}} \\
= & \frac{1}{2 i \pi} \sum_{i=1}^{s} \sum_{j=1}^{k-2} \sum_{I \in K_{j}} \oint_{\left[a_{2 i-1}, a_{2 i}\right]} \frac{F_{j+1}\left(x, x_{I}\right) F_{k-j}\left(x, x_{K-I}\right)}{2 M(x) \sigma(x)} d x \oint_{\left[a_{2 l-1}, a_{2 l}\right]} \frac{d x_{1}}{\left(x_{1}-x\right) \sqrt{\sigma\left(x_{1}\right)}} \\
& +\sum_{j=1}^{k-2} \sum_{I \in K_{j}} \oint_{\left[a_{2 l-1}, a_{2 l}\right]} \frac{F_{j+1}\left(x, x_{I}\right) F_{k-j}\left(x, x_{K-I}\right)}{2 M(x) \sqrt{\sigma(x)} \sigma(x)} d x
\end{aligned}
$$

Using the function $C_{l}(x)$ introduced in 3.24, we have:

$$
\begin{aligned}
& -\oint_{\left[a_{2 l-1}, a_{2 l}\right]} \frac{P_{k}\left(x_{1} ; x_{K}\right) d x_{1}}{\sqrt{\sigma\left(x_{1}\right)}} \\
= & \sum_{i=1}^{s} \sum_{j=1}^{k-2} \sum_{I \in K_{j}^{\prime}} \oint_{\left[a_{2 i-1}, a_{2 i}\right]} \frac{F_{j+1}\left(x, x_{I}\right) F_{k-j}\left(x, x_{K-I}\right)}{2 M(x) \sigma(x)} d x\left(C_{l}(x)+\frac{\delta_{i, l}}{\sqrt{\sigma(x)}}\right)
\end{aligned}
$$

and thus we have computed $P_{k}$ :

$$
\begin{aligned}
-P_{k}\left(x_{1} ; x_{K}\right)=\frac{1}{2 i \pi} \sum_{i=1}^{s} & \sum_{j=1}^{k-2} \sum_{I \in K_{j}} \oint_{\left[a_{2 i-1}, a_{2 i}\right]} \frac{F_{j+1}\left(x, x_{I}\right) F_{k-j}\left(x, x_{K-I}\right)}{2 M(x) \sigma(x)} d x \\
& \left(\frac{L_{i}\left(x_{1}\right)}{\sqrt{\sigma(x)}}+\sum_{l=1}^{s-1} C_{l}(x) L_{l}\left(x_{1}\right)\right)
\end{aligned}
$$

\subsection{The recursion relation}

That gives the recursion relation for the $F_{k}$ 's:

$$
\begin{aligned}
& F_{k}\left(x_{1} ; x_{K}\right)=\frac{1}{2 i \pi} \sum_{i=1}^{s} \sum_{j=1}^{k-2} \sum_{I \in K_{j}} \\
& \oint_{\left[a_{2 i-1}, a_{2 i}\right]} \frac{F_{j+1}\left(x, x_{I}\right) F_{k-j}\left(x, x_{K-I}\right)}{2 M(x) \sigma(x)} d x\left(\frac{1}{x_{1}-x}-\frac{L_{i}\left(x_{1}\right)}{\sqrt{\sigma(x)}}-\sum_{l=1}^{s-1} C_{l}(x) L_{l}\left(x_{1}\right)\right)
\end{aligned}
$$


where it is important to remember that the term inside the bracket is analytical when $x$ approaches $a_{2 i-1}$ or $a_{2 i}$. This allows to write the contour integrals as the sum of two residues around $a_{2 i-1}$ and $a_{2 i}$.

It is interesting and more intrinsic to rewrite 4.34 in terms of multi-linear differentials $G_{k}$ on the hyperelliptical curve.

First, notice that a contour around $a_{i}$ in the hyperelliptical curve $\left(\tau_{i}(x)=\sqrt{x-a_{i}}\right.$ around 0$)$ is twice the contour in the complex plane $\left(x\right.$ around $\left.a_{i}\right)$, i.e. we will have an extra factor 2 in the denominator.

Then, notice that $G_{2}$ and $F_{2}$ differ by a term, which is an even function of the local parameter $\tau_{i}(x)$, i.e. which does not contribute to residues near the branch-points (this can be checked separately for $k=3$ and $k>3$ ).

Thus we get the recursion relation for the $G_{k}$ 's:

$$
G_{k}\left(x_{1} ; x_{K}\right)=\sum_{i=1}^{2 s} \sum_{j=1}^{k-2} \sum_{I \in K_{j}} \operatorname{Res}_{a_{i} \in \Sigma} \frac{G_{j+1}\left(x, x_{I}\right) G_{k-j}\left(x, x_{K-I}\right)}{2 y(x) d x} d S_{i}\left(x_{1}, x\right)
$$

where now the residues are computed on the hyperelliptical surface (i.e. extra factor 2 in the denominator), and $d S_{i}\left(x, x^{\prime}\right)$ is the abelian differential of the third kind introduced in 3.26 .

That recursion relation allows to compute $W_{k}$ in a tree-like recursion from residues of lower loop-functions.

\subsection{Solution of the recursion relation as cubic-Feynmann trees}

Equation 4.35 is conveniently represented with diagrams "a la Feynmann".

Let us represent the $k$-loop correlation function $G_{k}\left(x_{1}, \ldots, x_{k}\right)$ as a black disk with $k$ legs:

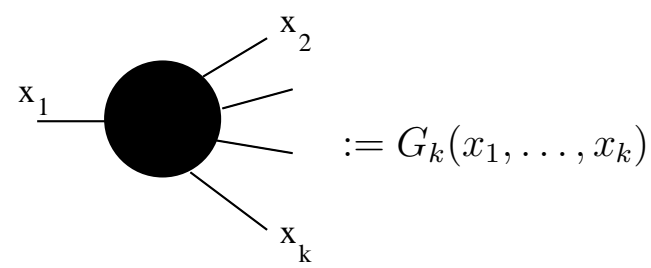

and introduce the following Feynmann rules: 


\begin{tabular}{|r|c|}
\hline Arrowed propagator: & $\mathrm{x}_{1} \longrightarrow \mathrm{x}_{2}:=d S_{i}\left(x, x^{\prime}\right)$ \\
\hline Vertex: & $\mathbf{X}:=1 /(2 y(x) d x)$ \\
\hline $\begin{array}{r}\text { Non-arrowed propagator } \\
=2-\text { loop correlator: }\end{array}$ & $\mathrm{x}_{1} \longrightarrow \mathrm{x}:={ }^{\mathrm{x}_{1}}$ \\
\hline
\end{tabular}

Then 4.35 can be represented as follows
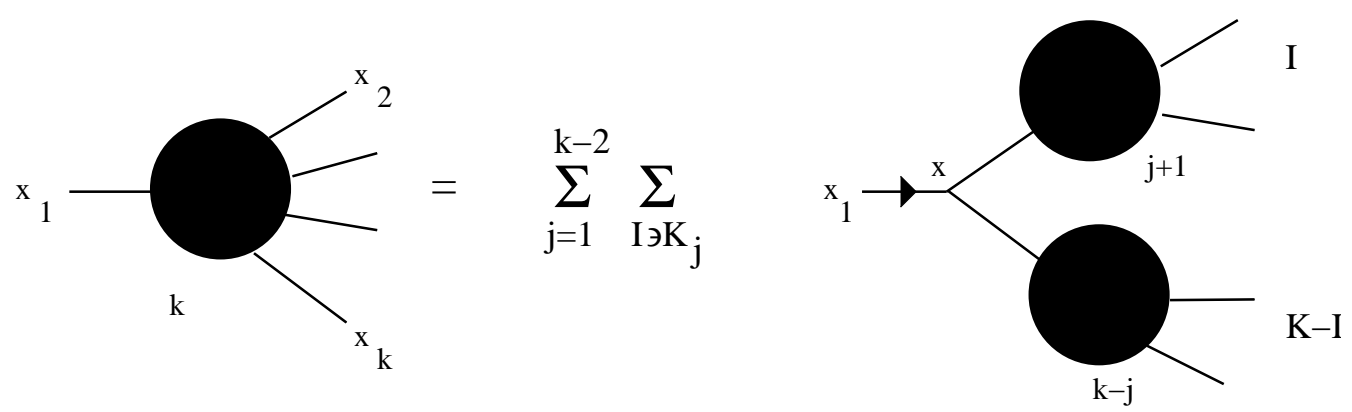

Whose solution is clearly that the $k$-loop correlator $G_{k}$ is the sum over all plane binary trees with 1 root and $k-1$ leaves, with a skeleton made of oriented arrows (oriented from root toward leaves), and whose $k-1$ leaves are non-arrowed propagators finishing at the $x_{j}$ 's with $2 \leq j \leq k$.

Notice that two trees which differ only by the ordering of branches at a vertex give the same contribution to $G_{k}$, so that instead of summing over plane trees, one can sum over non-plane trees, with a factor $2^{k-2}$.

Let $\mathcal{T}_{k}$ be the set of plane rooted binary trees with $k-1$ labeled leaves $\left(x_{2}, \ldots, x_{k}\right)$. and let $\overline{\mathcal{T}}_{k}$ be the set of non-plane rooted binary trees with $k-1$ labeled leaves $\left(x_{2}, \ldots, x_{k}\right)$. We have:

$$
N_{k+2}:=\operatorname{Card} \mathcal{T}_{k+2}=k+1 ! C_{k}=\frac{2 k !}{k !}=2^{k}(2 k-1) ! !
$$

where $C_{k}$ is the Catalan number which enumerates plane trees. And:

$$
\bar{N}_{k+2}:=\operatorname{Card} \overline{\mathcal{T}}_{k+2}=2^{-k} \operatorname{Card} \mathcal{T}_{k+2}=2^{-k} \frac{2 k !}{k !}=(2 k-1) ! !
$$

For any given tree $T \in \mathcal{T}_{k}$, with root $x_{1}$, leaves $x_{j}(j=2, \ldots, k)$, and with $k-2$ vertices noted $x_{v}^{\prime}(v=1, \ldots, k-2)$, so that its inner edges are of the form $v_{1} \rightarrow v_{2}$ and its outer edges are of the form $v \rightarrow j$, we define the weight of $T$ as:

$$
\begin{aligned}
\mathcal{W}(T):= & \prod_{\text {vertex } v=1}^{k-2} \sum_{i_{v}=1}^{2 s} \prod_{x_{v}^{\prime} \rightarrow a_{i}}^{\operatorname{Res}} \frac{1}{2 y\left(x_{v}^{\prime}\right) d x_{v}^{\prime}} d S_{i_{v}}\left(x_{v}^{\prime}, x_{w}^{\prime}\right) \prod_{\text {inner edges } v \rightarrow w} G_{2}\left(x_{v}^{\prime}, x_{j}\right)
\end{aligned}
$$


Thus we have:

$$
G_{k}\left(x_{1}, \ldots, x_{k}\right)=2^{k-2} \sum_{T \in \overline{\mathcal{T}}_{k}} \mathcal{W}(T)=\sum_{T \in \mathcal{T}_{k}} \mathcal{W}(T)
$$

$G_{3}$ is thus given by $\bar{N}_{3}=1$ tree, $G_{4}$ is the sum of $\bar{N}_{4}=3$ diagrams, $G_{5}$ is the sum of $\bar{N}_{5}=15$ diagrams, ...

\subsection{Example: 3-point function}

As an example, let us carry out explicitly the computation for the 3-point function.

Diagrammatically, we have:
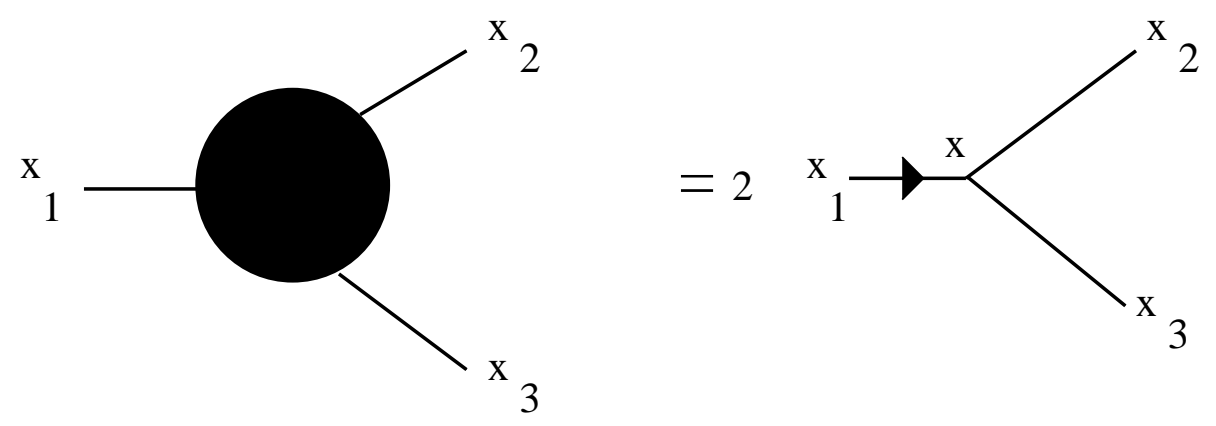

Eq4.28 can be written for $k=3$ :

$$
F_{3}\left(x_{1}, x_{2}, x_{3}\right)=\sum_{i=1}^{2 s} \operatorname{Res}_{a_{i}} d x \frac{F_{2}\left(x, x_{2}\right) F_{2}\left(x, x_{3}\right)}{M(x) \sigma(x) \sigma^{\prime}(x)} \frac{\sigma^{\prime}(x)}{\left(x-x_{1}\right)}+P_{3}\left(x_{1}, x_{2}, x_{3}\right)
$$

where $P_{3}$ is a polynomial in $x_{1}$. Using 4.18, notice that $F_{2}\left(a_{i}, x\right)=\frac{\sigma^{\prime}\left(a_{i}\right)}{x-a_{i}}+A\left(a_{i}, x\right)$ is finite, and that:

$$
\frac{\sigma^{\prime}(x)}{x-x_{1}}=2 \frac{\sigma(x)}{\left(x-x_{1}\right)^{2}}-F_{2}\left(x, x_{1}\right)+A\left(x, x_{1}\right)
$$

i.e.

$$
\begin{aligned}
F_{3}\left(x_{1}, x_{2}, x_{3}\right)= & 2 \sum_{i=1}^{2 s} \operatorname{Res}_{a_{i}} d x \frac{F_{2}\left(x, x_{2}\right) F_{2}\left(x, x_{3}\right)}{M(x)\left(x-x_{1}\right)^{2} \sigma^{\prime}(x)} \\
& -\sum_{i=1}^{2 s} \operatorname{Res}_{a_{i}} d x \frac{F_{2}\left(x, x_{1}\right) F_{2}\left(x, x_{2}\right) F_{2}\left(x, x_{3}\right)}{M(x) \sigma(x) \sigma^{\prime}(x)} \\
& +\sum_{i=1}^{2 s} \operatorname{Res}_{a_{i}} d x \frac{A\left(x, x_{1}\right) F_{2}\left(x, x_{2}\right) F_{2}\left(x, x_{3}\right)}{M(x) \sigma(x) \sigma^{\prime}(x)}+R_{3}\left(x_{1}, x_{2}, x_{3}\right)
\end{aligned}
$$


The first line has no residue at the branch-points (indeed, using 4.18, notice that $F_{2}\left(a_{i}, x\right)$ is finite), and the last line is a polynomial in $x_{1}$ (indeed $A\left(x, x_{1}\right)$ is), therefore:

$$
\begin{aligned}
F_{3}\left(x_{1}, x_{2}, x_{3}\right) & =-\sum_{i=1}^{2 s} \operatorname{Res}_{a_{i}} d x \frac{F_{2}\left(x, x_{1}\right) F_{2}\left(x, x_{2}\right) F_{2}\left(x, x_{3}\right)}{M(x) \sigma(x) \sigma^{\prime}(x)}+\tilde{R}_{3}\left(x_{1}, x_{2}, x_{3}\right) \\
& =-\sum_{i=1}^{2 s} \frac{F_{2}\left(a_{i}, x_{1}\right) F_{2}\left(a_{i}, x_{2}\right) F_{2}\left(a_{i}, x_{3}\right)}{M\left(a_{i}\right) \sigma^{\prime}\left(a_{i}\right)^{2}}+\tilde{R}_{3}\left(x_{1}, x_{2}, x_{3}\right)
\end{aligned}
$$

where $\tilde{R}_{3}\left(x_{1}, x_{2}, x_{3}\right)$ is a polynomial in $x_{1}$, of degree at most $s-2$. Condition 2.12 implies that $\tilde{R}_{3}\left(x_{1}, x_{2}, x_{3}\right)=0$, thus:

$$
F_{3}\left(x_{1}, x_{2}, x_{3}\right)=-\sum_{i=1}^{2 s} \frac{F_{2}\left(a_{i}, x_{1}\right) F_{2}\left(a_{i}, x_{2}\right) F_{2}\left(a_{i}, x_{3}\right)}{M\left(a_{i}\right) \sigma^{\prime}\left(a_{i}\right)^{2}}
$$

This is a generalization of what was found in [1, 2, 3] for the one-cut case $s=1$.

Let us redo this computation in a more intrinsic way. Start from 4.35 for $k=3$ :

$$
G_{3}\left(x_{1}, x_{2}, x_{3}\right)=2 \sum_{i=1}^{2 s} \operatorname{Res}_{a_{i}} \frac{G_{2}\left(x, x_{2}\right) G_{2}\left(x, x_{3}\right)}{2 y(x) d x} d S_{i}\left(x_{1}, x\right)
$$

Notice that both $d S_{i}\left(x_{1}, x\right)$ and $y(x)$ have a simple zero at $x=a_{i}$ thus,

$$
\begin{aligned}
\frac{d S_{i}\left(x_{1}, x\right)}{y(x)} & =\frac{d_{x} d S_{i}\left(x_{1}, x\right)}{d y(x)}+O\left(\sqrt{x-a_{i}}\right) \\
& =\frac{B\left(x_{1}, x\right)+B\left(x_{1}, \bar{x}\right)}{d y(x)}+O\left(\sqrt{x-a_{i}}\right) \\
& =2 \frac{G_{2}\left(x_{1}, x\right)}{d y(x)}+O\left(\sqrt{x-a_{i}}\right)
\end{aligned}
$$

This implies:

$$
G_{3}\left(x_{1}, x_{2}, x_{3}\right)=2 \sum_{i=1}^{2 s} \operatorname{Res}_{a_{i}} \frac{G_{2}\left(x, x_{2}\right) G_{2}\left(x, x_{3}\right) G_{2}\left(x, x_{1}\right)}{d x d y}
$$

This agrees with [29, 5] (our $d y$ is half the $d y$ of [5]). One can also write:

$$
W_{3}\left(x_{1}, x_{2}, x_{3}\right)=2 \sum_{i=1}^{2 s} \operatorname{Res}_{a_{i}} W_{2}\left(x, x_{2}\right) W_{2}\left(x, x_{3}\right) W_{2}\left(x, x_{1}\right) \frac{d x^{2}}{d y}
$$




\subsection{Example: 4 point function}

Diagrammatically, we have:

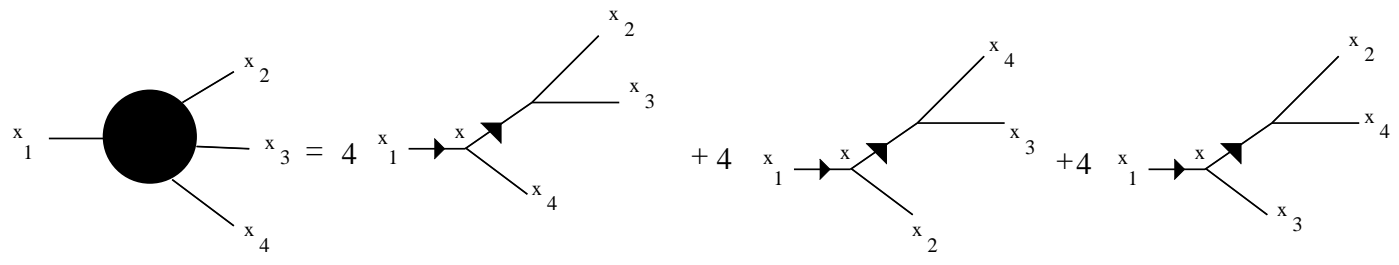

Explicit computation of 4.34 for $k=4$ gives:

$$
\begin{aligned}
F_{4}\left(x_{1}, x_{2}, x_{3}, x_{4}\right)= & -\sum_{i \neq j=1}^{2 s} \frac{F_{2}\left(x_{3}, a_{j}\right) F_{2}\left(x_{4}, a_{j}\right)}{M\left(a_{j}\right) \sigma^{\prime}\left(a_{j}\right)^{2}} F_{2}\left(a_{i}, a_{j}\right) \frac{F_{2}\left(x_{2}, a_{i}\right) F_{2}\left(x_{1}, a_{i}\right)}{M\left(a_{i}\right) \sigma^{\prime}\left(a_{i}\right)^{2}} \\
& -\sum_{i \neq j=1}^{2 s} \frac{F_{2}\left(x_{3}, a_{j}\right) F_{2}\left(x_{2}, a_{j}\right)}{M\left(a_{j}\right) \sigma^{\prime}\left(a_{j}\right)^{2}} F_{2}\left(a_{i}, a_{j}\right) \frac{F_{2}\left(x_{4}, a_{i}\right) F_{2}\left(x_{1}, a_{i}\right)}{M\left(a_{i}\right) \sigma^{\prime}\left(a_{i}\right)^{2}} \\
& -\sum_{i \neq j=1}^{2 s} \frac{F_{2}\left(x_{2}, a_{j}\right) F_{2}\left(x_{4}, a_{j}\right)}{M\left(a_{j}\right) \sigma^{\prime}\left(a_{j}\right)^{2}} F_{2}\left(a_{i}, a_{j}\right) \frac{F_{2}\left(x_{3}, a_{i}\right) F_{2}\left(x_{1}, a_{i}\right)}{M\left(a_{i}\right) \sigma^{\prime}\left(a_{i}\right)^{2}} \\
& -\sum_{i=1}^{2 s} \frac{\left(F_{2}\left(x_{3}, a_{i}\right) F_{2}\left(x_{4}, a_{i}\right) F_{2}\left(a_{i}, x_{2}\right) F_{2}\left(a_{i}, x_{1}\right)\right)^{\prime}}{M\left(a_{i}\right)^{2} \sigma^{\prime}\left(a_{i}\right)^{3}} \\
& +3 \sum_{i=1}^{2 s} \frac{F_{2}\left(x_{3}, a_{i}\right) F_{2}\left(x_{4}, a_{i}\right) F_{2}\left(a_{i}, x_{2}\right) F_{2}\left(a_{i}, x_{1}\right)}{M\left(a_{i}\right)^{2} \sigma^{\prime}\left(a_{i}\right)^{3}} \\
& \left(\frac{M^{\prime}\left(a_{i}\right)}{M\left(a_{i}\right)}-\frac{A\left(a_{i}, a_{i}\right)}{\sigma^{\prime}\left(a_{i}\right)}+\frac{5 \sigma^{\prime \prime}\left(a_{i}\right)}{6 \sigma^{\prime}\left(a_{i}\right)}\right)
\end{aligned}
$$

\section{$5 \quad$ Higher genus}

Now, we don't drop the $1 / N^{2}$ term in 4.6, and we expand to order $h$ :

$$
\begin{aligned}
& 2 \sum_{m=0}^{h} W_{1}^{(h-m)}\left(x_{1}\right) W_{k}^{(m)}\left(x_{1}, \ldots, x_{k}\right) \\
& +W_{k+1}^{(h-1)}\left(x_{1}, x_{1}, x_{2}, \ldots, x_{k}\right) \\
& +\sum_{m=0}^{h} \sum_{j=1}^{k-2} \sum_{I \in K_{j}} W_{j+1}^{(m)}\left(x_{1}, x_{I}\right) W_{k-j}^{(h-m)}\left(x_{1}, x_{K-I}\right) \\
& +\sum_{j=2}^{k} \frac{\partial}{\partial x_{j}} \frac{W_{k-1}^{(h)}\left(x_{2}, \ldots, x_{j}, \ldots, x_{k}\right)-W_{k-1}^{(h)}\left(x_{2}, \ldots, x_{1}, \ldots, x_{k}\right)}{x_{j}-x_{1}} \\
& =V^{\prime}\left(x_{1}\right) W_{k}^{(h)}\left(x_{1}, \ldots, x_{k}\right)-U_{k}^{(h)}\left(x_{1} ; x_{2}, \ldots, x_{k}\right)
\end{aligned}
$$

thus:

$$
M\left(x_{1}\right) \sqrt{\sigma\left(x_{1}\right)} W_{k}^{(h)}\left(x_{1}, x_{K}\right)
$$




$$
\begin{aligned}
& =2 \sum_{m=0}^{h-1} W_{1}^{(h-m)}\left(x_{1}\right) W_{k}^{(m)}\left(x_{1}, x_{K}\right)+W_{k+1}^{(h-1)}\left(x_{1}, x_{1}, x_{K}\right) \\
& +\sum_{m=0}^{h} \sum_{j=1}^{k-2} \sum_{I \in K_{j}} W_{j+1}^{(m)}\left(x_{1}, x_{I}\right) W_{k-j}^{(h-m)}\left(x_{1}, x_{K-I}\right) \\
& +\sum_{j=2}^{k} \frac{\partial}{\partial x_{j}} \frac{W_{k-1}^{(h)}\left(x_{K}\right)-W_{k-1}^{(h)}\left(x_{1}, x_{K-\{j\}}\right)}{x_{j}-x_{1}}+U_{k}^{(h)}\left(x_{1} ; x_{K}\right)
\end{aligned}
$$

In particular for $k=1,5.2$ reads :

$$
M\left(x_{1}\right) \sqrt{\sigma\left(x_{1}\right)} W_{1}^{(h)}\left(x_{1}\right)=\sum_{m=1}^{h-1} W_{1}^{(h-m)}\left(x_{1}\right) W_{1}^{(m)}\left(x_{1}\right)+W_{2}^{(h-1)}\left(x_{1}, x_{1}\right)+U_{1}^{(h)}\left(x_{1}\right)
$$

It is easy to prove, by double recursion on $k$ and $h$, that

$$
F_{k}^{(h)}\left(x_{1}, \ldots, x_{k}\right)=2^{k} W_{k}^{(h)}\left(x_{1}, \ldots, x_{k}\right) \prod_{i=1}^{k} \sqrt{\sigma\left(x_{i}\right)}
$$

is a rational function. Introduce the Euclidean division of the polynomial $U_{k}^{(h)}\left(x_{1} ; x_{K}\right)$ by $M\left(x_{1}\right)$ :

$$
U_{k}^{(h)}\left(x_{1} ; x_{K}\right)=\frac{2^{-k}}{\sqrt{\sigma\left(x_{K}\right)}} P_{k}^{(h)}\left(x_{1} ; x_{K}\right) M\left(x_{1}\right)+Q_{k}^{(h)}\left(x_{1} ; x_{K}\right)
$$

where $\operatorname{deg} P_{k}^{(h)}=s-2$ and $\operatorname{deg} Q_{k}^{(h)}<d-s$. 5.2 becomes:

$$
\begin{aligned}
& \sqrt{\sigma\left(x_{1}\right)} W_{k}^{(h)}\left(x_{1}, x_{K}\right)-\frac{2^{-k}}{\sqrt{\sigma\left(x_{K}\right)}} P_{k}^{(h)}\left(x_{1} ; x_{K}\right) \\
& =2 \sum_{m=0}^{h-1} \frac{W_{1}^{(h-m)}\left(x_{1}\right) W_{k}^{(m)}\left(x_{1}, x_{K}\right)}{M\left(x_{1}\right)}+\frac{W_{k+1}^{(h-1)}\left(x_{1}, x_{1}, x_{K}\right)}{M\left(x_{1}\right)} \\
& +\sum_{m=0}^{h} \sum_{j=1}^{k-2} \sum_{I \in K_{j}} \frac{W_{j+1}^{(m)}\left(x_{1}, x_{I}\right) W_{k-j}^{(h-m)}\left(x_{1}, x_{K-I}\right)}{M\left(x_{1}\right)} \\
& +\sum_{j=2}^{k} \frac{\partial}{\partial x_{j}} \frac{W_{k-1}^{(h)}\left(x_{K}\right)-W_{k-1}^{(h)}\left(x_{1}, x_{K-\{j\}}\right)}{\left(x_{j}-x_{1}\right) M\left(x_{1}\right)}+Q_{k}^{(h)}\left(x_{1} ; x_{K}\right)
\end{aligned}
$$

where the LHS is a rational function of $x_{1}$ with poles only at the branch points, therefore:

$$
\sqrt{\sigma\left(x_{1}\right)} W_{k}^{(h)}\left(x_{1}, x_{K}\right)-\frac{2^{-k}}{\sqrt{\sigma\left(x_{K}\right)}} P_{k}^{(h)}\left(x_{1} ; x_{K}\right)
$$




$$
\begin{aligned}
& =\operatorname{Res}_{x \rightarrow x_{1}} \frac{d x}{x-x_{1}}\left(\sqrt{\sigma(x)} W_{k}^{(h)}\left(x, x_{K}\right)-P_{k}^{(h)}\left(x ; x_{K}\right)\right) \\
& =\sum_{i=1}^{2 s} \operatorname{Res}_{x \rightarrow a_{i}} \frac{d x}{x_{1}-x}\left(\sqrt{\sigma(x)} W_{k}^{(h)}\left(x, x_{K}\right)-P_{k}^{(h)}\left(x ; x_{K}\right)\right) \\
& =\sum_{i=1}^{2 s} \operatorname{Res}_{x \rightarrow a_{i}} \frac{d x}{x_{1}-x}\left(2 \sum_{m=0}^{h-1} \frac{W_{1}^{(h-m)}(x) W_{k}^{(m)}\left(x, x_{K}\right)}{M(x)}+\frac{W_{k+1}^{(h-1)}\left(x, x, x_{K}\right)}{M(x)}\right) \\
& +\sum_{i=1}^{2 s} \operatorname{Res}_{x \rightarrow a_{i}} \frac{d x}{x_{1}-x}\left(\sum_{m=0}^{h} \sum_{j=1}^{k-2} \sum_{I \in K_{j}} \frac{W_{j+1}^{(m)}\left(x, x_{I}\right) W_{k-j}^{(h-m)}\left(x, x_{K-I}\right)}{M(x)}\right) \\
& +\sum_{i=1}^{2 s} \operatorname{Res}_{x \rightarrow a_{i}} \frac{d x}{x_{1}-x}\left(\sum_{j=2}^{k} \frac{\partial}{\partial x_{j}} \frac{W_{k-1}^{(h)}\left(x_{K}\right)-W_{k-1}^{(h)}\left(x, x_{K-\{j\}}\right)}{\left(x_{j}-x\right) M(x)}+\frac{Q_{k}^{(h)}\left(x ; x_{K}\right)}{M(x)}\right)
\end{aligned}
$$

Two terms in the last line have no pole at $x_{1}=a_{i}$, and the other term in the last line combines with other lines so as to transform $W_{2}$ in $F_{2}$. Thus we get:

$$
\begin{aligned}
F_{k}^{(h)}\left(x_{1}, x_{K}\right)= & \frac{1}{2} \sum_{i=1}^{2 s} \operatorname{Res}_{x \rightarrow a_{i}} \frac{d x}{x_{1}-x} \sum_{m=0}^{h} \sum_{j=0}^{k-1}\left(1-\delta_{m, 0} \delta_{j, 0}-\delta_{m, h} \delta_{j, k-1}\right) \\
& \sum_{I \in K_{j}} \frac{F_{j+1}^{(m)}\left(x, x_{I}\right) F_{k-j}^{(h-m)}\left(x, x_{K-I}\right)}{M(x) \sigma(x)} \\
& +\frac{1}{2} \sum_{i=1}^{2 s} \operatorname{Res}_{x \rightarrow a_{i}} \frac{d x}{x_{1}-x} \frac{F_{k+1}^{(h-1)}\left(x, x, x_{K}\right)}{M(x) \sigma(x)} \\
& +P_{k}^{(h)}\left(x_{1} ; x_{K}\right)
\end{aligned}
$$

where $P_{k}^{(h)}\left(x_{1} ; x_{K}\right)$ is obtained from 3.19 in a way very similar to what we have done to leading order. Finally we find:

$$
\begin{aligned}
G_{k}^{(h)}\left(x_{1}, x_{K}\right)= & \sum_{i=1}^{2 s} \operatorname{Res}_{x \rightarrow a_{i}} d S_{i}\left(x_{1}, x\right) \frac{G_{k+1}^{(h-1)}\left(x, x, x_{K}\right)}{y(x) d x} \\
& +2 \sum_{i=1}^{2 s} \operatorname{Res}_{x \rightarrow a_{i}} \sum_{m=0}^{h-1} d S_{i}\left(x_{1}, x\right) \frac{G_{1}^{(h-m)}(x) G_{k}^{(m)}\left(x, x_{K}\right)}{y(x) d x} \\
& +\sum_{i=1}^{2 s} \operatorname{Res}_{x \rightarrow a_{i}} \sum_{m=0}^{h} \sum_{j=1}^{k-2} \sum_{I \in K_{j}} \\
& \quad d S_{i}\left(x_{1}, x\right) \frac{G_{j+1}^{(m)}\left(x, x_{I}\right) G_{k-j}^{(h-m)}\left(x, x_{K-I}\right)}{y(x) d x}
\end{aligned}
$$

where one should notice that the first line correspond to $j=0$ and $j=k-1$ in the second line.

Let us represent the order $N^{-2 h} k$-loop correlation function $G_{k}^{(h)}\left(x_{1}, \ldots, x_{k}\right)$ as a 

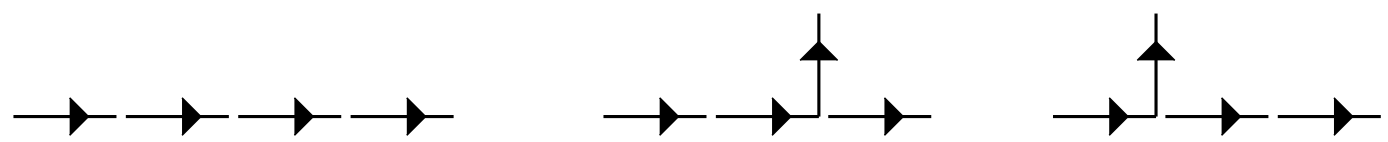

Figure 1: The 3 skeleton trees contributing to $\mathcal{T}_{2}^{(2)}$, i.e. with $k+2 h-2=4$ edges.

black disk with $k$ legs and $h$ holes:

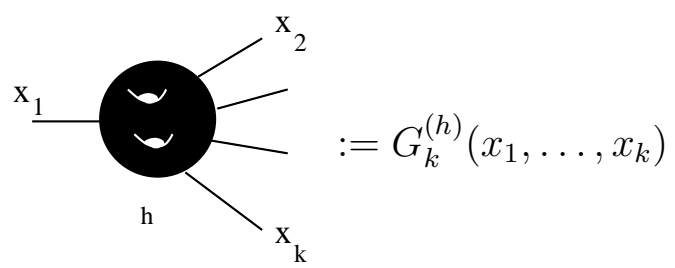

Using the Feynmann rules introduced above, 5.9 can be represented as:

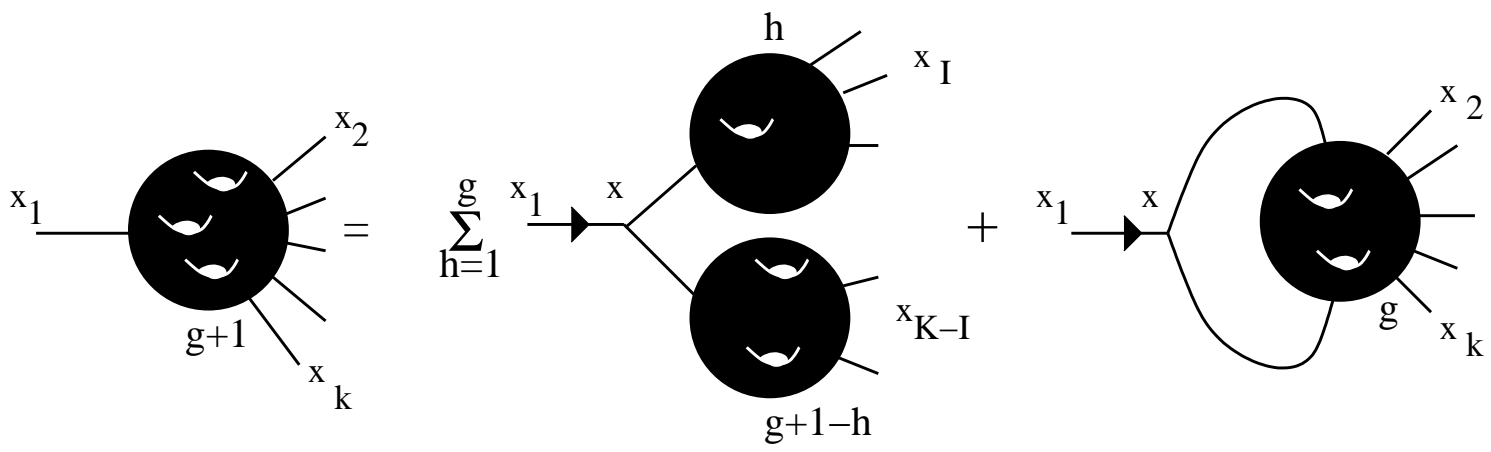

which means that $G_{k}^{(h)}$ is obtained by summing over all Feynmann graphs with $k$ external legs and $h$ loops.

The set $\mathcal{T}_{k}^{(h)}$ of all possible graphs with $k$ external legs, and with $h$ loops, can be described as follows: First, draw all rooted skeleton trees (i.e. trees whose vertices have valence 1,2 or 3 ), containing $k+2 h-2$ edges. Draw arrows on the edges, oriented from root toward leaves (see fig 1). Then draw, in all possible ways, $k-1$ external legs, and $h$ inner edges, with the constraint that all the vertices of the whole graph have valence 3, and so that an inner edge can be drawn only between a vertex and one of its descendents (inner edges can never connect different branches of the tree), see fig 2 for the example $k=2, h=2$. Then, each such graph has a symmetry factor.

We have (see appendix 71):

$$
N_{k}^{(h)}:=\operatorname{Card} \mathcal{T}_{k}^{(h)}=s_{h}(k-1) ! 4^{k-1}\left(\begin{array}{c}
\frac{3(h-1)}{2}+k-1 \\
k-1
\end{array}\right)
$$

where $s_{h}=N_{1}^{(h)}$ is the number of one-leg graphs in a usual $\phi^{3}$ field theory. The generating function $s(x)=\sum_{h=1}^{\infty} s_{h} x^{h-1}$ is computed in appendix 7 in terms of Airy 

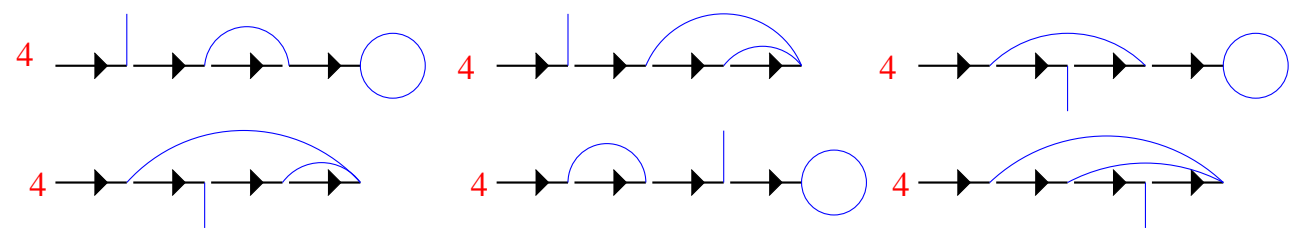

4
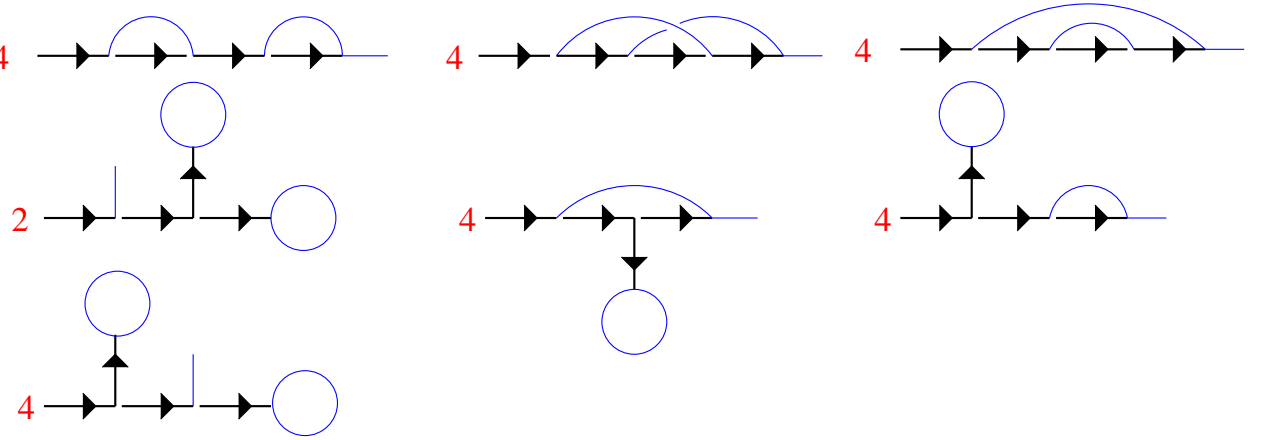

4

Figure 2: All the possible ways of drawing $k-1=1$ external leg and $h=2$ inner edges, so that the graphs are trivalent, and that inner edges never connect different branches. Notice that all but one graph have symmetry factor 4, and one has 2 .

function. We have:

$$
s_{1}=1 \quad, \quad s_{2}=5 \quad, \quad s_{3}=60 \quad, \quad \ldots
$$

In particular for genus $h=1$ :

$$
N_{k}^{(1)}:=\operatorname{Card} \mathcal{T}_{k}^{(1)}=4^{k-1}(k-1) !
$$

Similarly to 4.40

$$
G_{k}^{(h)}\left(x_{1}, \ldots, x_{k}\right)=\sum_{T \in \mathcal{T}_{k}^{(h)}} \mathcal{W}(T)
$$

where the weight $\mathcal{W}$ was defined in 4.39

\subsection{Example: One-loop function, genus one}

Let us carry out explicitly the case $k=1, h=1$, and recover the result of [16, 17, 15]: In that case, 5.1 reads:

$$
\sqrt{\sigma\left(x_{1}\right)} W_{1}^{(1)}\left(x_{1}\right)=\frac{W_{2}\left(x_{1}, x_{1}\right)+U_{1}^{(1)}\left(x_{1}\right)}{M\left(x_{1}\right)}
$$

The RHS is clearly a rational function of $x_{1}$, and from 2.14 we know that the LHS has poles only at the branch-points, and at $\infty$. Introduce the Euclidean division of the 
polynomial $U_{1}^{(h)}\left(x_{1}\right)$ by $M\left(x_{1}\right)$ :

$$
U_{1}^{(1)}\left(x_{1}\right)=P_{1}^{(1)}\left(x_{1}\right) M\left(x_{1}\right)+Q_{1}^{(1)}\left(x_{1}\right)
$$

where $\operatorname{deg} P_{1}^{(1)}=s-2$ and $\operatorname{deg} Q_{1}^{(1)}<d-s$.

We may thus write:

$$
\begin{aligned}
\sqrt{\sigma\left(x_{1}\right)} W_{1}^{(1)}\left(x_{1}\right)-P_{1}^{(1)}\left(x_{1}\right) & =\operatorname{Res}_{x \rightarrow x_{1}} \frac{d x}{x-x_{1}}\left(\sqrt{\sigma(x)} W_{k}^{(1)}(x)-P_{1}^{(1)}(x)\right) \\
& =\sum_{i=1}^{2 s} \operatorname{Res}_{x \rightarrow a_{i}} \frac{d x}{x_{1}-x}\left(\sqrt{\sigma(x)} W_{k}^{(1)}(x)-P_{1}^{(1)}(x)\right) \\
& =\sum_{i=1}^{2 s} \operatorname{Res}_{x \rightarrow a_{i}} \frac{d x}{x_{1}-x} \frac{W_{2}(x, x)+Q_{1}^{(1)}(x)}{M(x)} \\
& =\sum_{i=1}^{2 s} \operatorname{Res}_{x \rightarrow a_{i}} \frac{d x}{x_{1}-x} \frac{W_{2}(x, x)}{M(x)}
\end{aligned}
$$

It clearly gives:

$$
G_{1}^{(1)}\left(x_{1}\right)=\sum_{i=1}^{2 s} \operatorname{Res}_{x \rightarrow a_{i}} \frac{G_{2}(x, x)}{y(x) d x} d S_{i}\left(x_{1}, x\right)
$$

Diagrammatically we have:

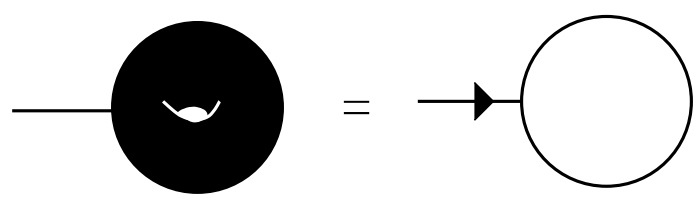

One can check that this result is identical to the function $W_{1}^{(1)}(x)$ computed in [15].

Similarly we have:
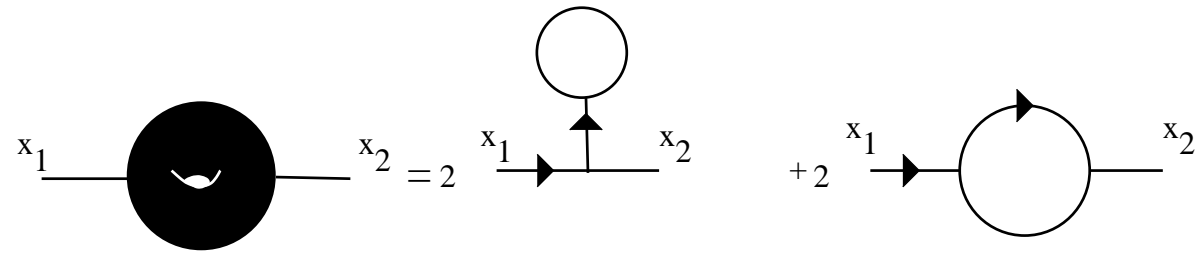

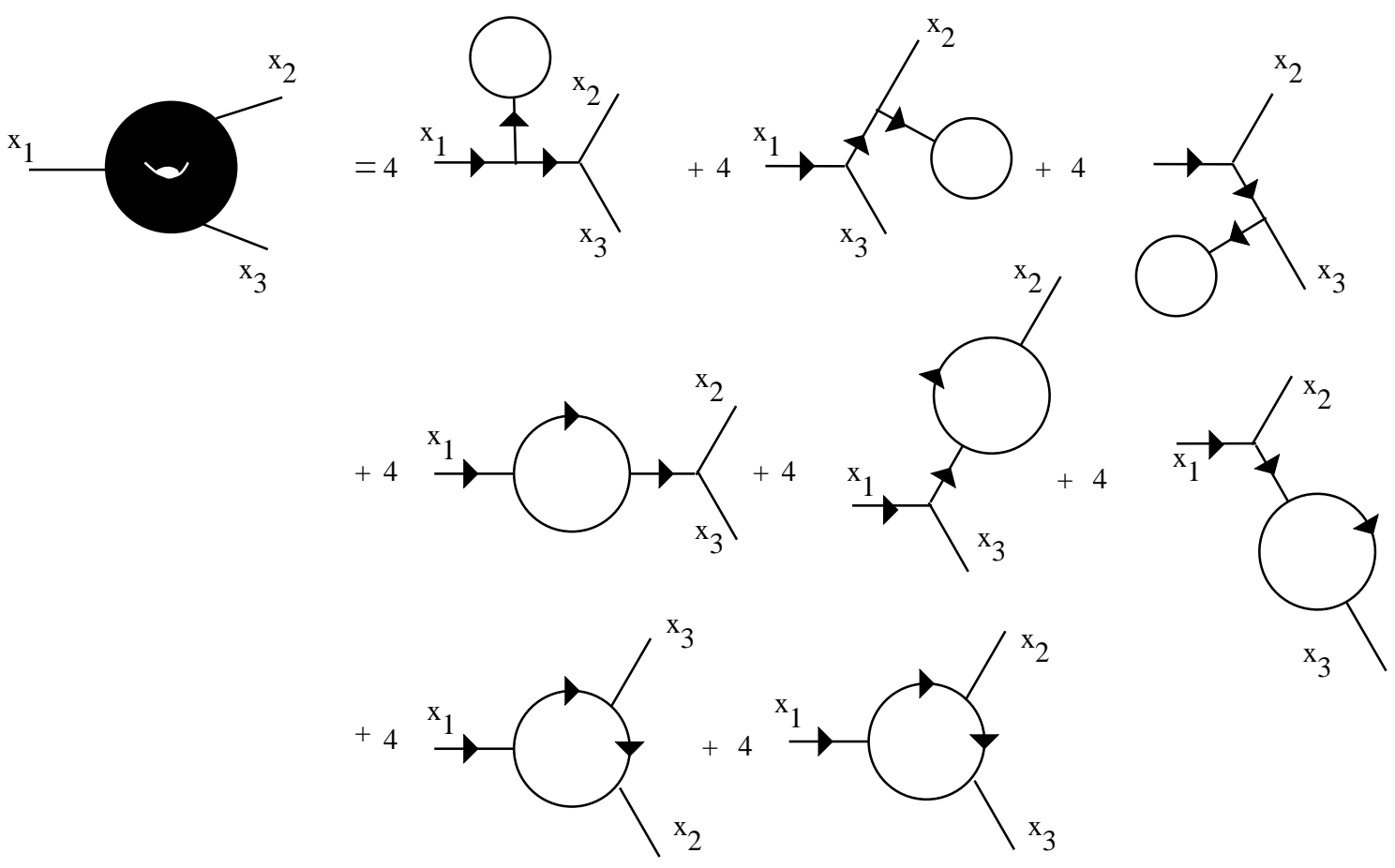

and at genus 2 we have:

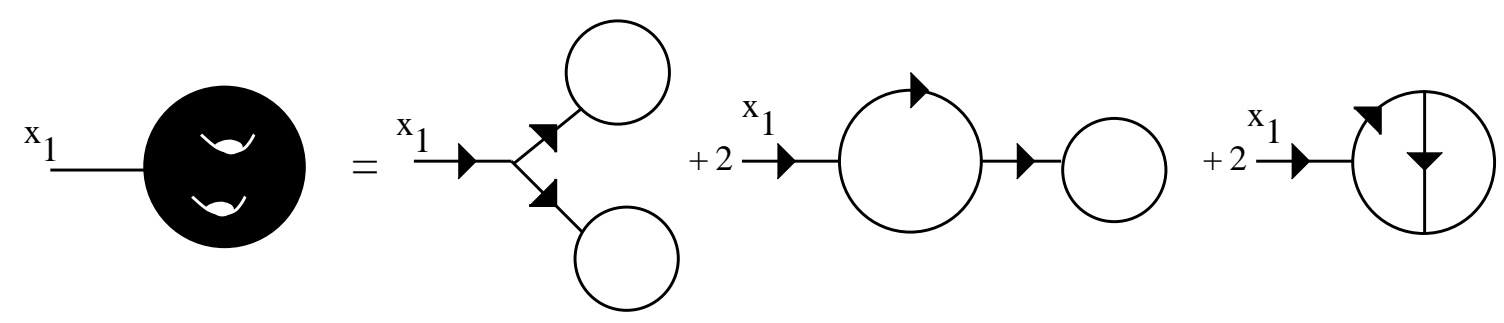

and so on...

\section{Example, One-cut case $s=1$, i.e. genus zero curve}

We write:

$$
\sigma(x)=(x-a)(x-b)
$$

It is convenient to map the genus zero hyperelliptical surface into the complex plane with the rational map:

$$
\begin{gathered}
x(\lambda)=\frac{a+b}{2}+\gamma\left(\lambda+\lambda^{-1}\right) \\
\gamma=\frac{b-a}{4}
\end{gathered}
$$


The $x$-physical sheet is sent to the exterior of the unique disc in the $\lambda$-plane, and the $x$-second sheet is sent to the interior of the unique disc in the $\lambda$-plane. We have:

$$
\sqrt{\sigma(x(\lambda))}=\gamma\left(\lambda-\lambda^{-1}\right)
$$

With this parameterization, all correlation functions are rational functions of the $\lambda$ 's.

\subsection{Recursion relations}

All $P_{k}$ 's are identically vanishing. We have the formula:

$$
\begin{aligned}
F_{2}\left(x_{1}, x_{2}\right) & =2 \sqrt{\sigma\left(x_{2}\right)} \frac{\partial}{\partial x_{2}} \frac{\sqrt{\sigma\left(x_{2}\right)}}{\left(x_{1}-x_{2}\right)}=\frac{2 x_{1} x_{2}-(a+b)\left(x_{1}+x_{2}\right)+2 a b}{\left(x_{1}-x_{2}\right)^{2}} \\
F_{k}\left(x_{1}, \ldots, x_{k}\right) & =-\frac{1}{2} \operatorname{Res}_{a_{i}}\left(\sum_{j=1}^{k-2} \sum_{I \in K_{j}} \frac{F_{j+1}\left(x, x_{I}\right) F_{k-j}\left(x, x_{K-I}\right)}{\left(x-x_{1}\right) M(x) \sigma(x)}\right) d x \text { for } k \geq 3
\end{aligned}
$$

and for $k+h>1,5.9$ gives:

$$
\begin{aligned}
F_{k}^{(h)}\left(x_{1}, x_{K}\right)= & 2 \sum_{i=1}^{2 s} \operatorname{Res}_{x \rightarrow a_{i}} \sum_{m=0}^{h-1} \frac{F_{1}^{(h-m)}(x) F_{k}^{(m)}\left(x, x_{K}\right)}{\left(x_{1}-x\right) M(x) \sigma(x)} d x \\
& +\sum_{i=1}^{2 s} \operatorname{Res}_{x \rightarrow a_{i}} \sum_{m=0}^{h} \sum_{j=1}^{k-2} \sum_{I \in K_{j}} \frac{F_{j+1}^{(m)}\left(x, x_{I}\right) F_{k-j}^{(h-m)}\left(x, x_{K-I}\right)}{\left(x_{1}-x\right) M(x) \sigma(x)} d x \\
& +\sum_{i=1}^{2 s} \operatorname{Res}_{x \rightarrow a_{i}} \frac{F_{k+1}^{(h-1)}\left(x, x, x_{K}\right)}{\left(x_{1}-x\right) M(x) \sigma(x)} d x
\end{aligned}
$$

\subsection{2 point function}

The 2-point function can be written as:

$$
W_{2}\left(x_{1}, x_{2}\right)=-\frac{\partial}{\partial x_{1}} \frac{\partial}{\partial x_{2}} \ln \left(\lambda_{1}-\lambda_{2}^{-1}\right)=\frac{\partial}{\partial x_{1}} \frac{\partial}{\partial x_{2}} \ln \left(\frac{\lambda_{1}-\lambda_{2}}{x_{1}-x_{2}}\right)
$$

where

$$
x_{1}=\gamma\left(\lambda_{1}+\lambda_{1}^{-1}\right) \quad, \quad x_{2}=\gamma\left(\lambda_{2}+\lambda_{2}^{-1}\right)
$$

or:

$$
\begin{aligned}
W_{2}\left(x_{1}, x_{2}\right) & =-\frac{1}{4 \sqrt{\sigma\left(x_{1}\right)} \sqrt{\sigma\left(x_{2}\right)}}\left(1-\left(\frac{\sqrt{\sigma\left(x_{1}\right)}-\sqrt{\sigma\left(x_{2}\right)}}{x_{1}-x_{2}}\right)^{2}\right) \\
& =-\frac{1}{2\left(x_{1}-x_{2}\right)^{2}}+\frac{2 x_{1} x_{2}-(a+b)\left(x_{1}+x_{2}\right)+2 a b}{4\left(x_{1}-x_{2}\right)^{2} \sqrt{\sigma\left(x_{1}\right)} \sqrt{\sigma\left(x_{2}\right)}}
\end{aligned}
$$


In particular we have:

$$
F_{2}(a, x)=\frac{(a-b)}{(x-a)} \quad, \quad F_{2}(b, x)=\frac{(b-a)}{(x-b)}
$$

The polynomial $A\left(x_{1}, x_{2}\right)$ introduced in 3.31 vanishes identically, and we have:

$$
W_{2}(x, x)=\frac{(b-a)^{2}}{16 \sigma(x)^{2}}
$$

All this is well known, see for instance [1].

\subsection{Other correlation functions}

We just give some examples of applications of the general theory described above:

$$
\begin{aligned}
F_{3}\left(x_{1}, x_{2}, x_{3}\right)= & -\operatorname{Res}_{a, b}\left(\frac{F_{2}\left(x, x_{2}\right) F_{2}\left(x, x_{3}\right)}{\left(x-x_{1}\right) M(x) \sigma(x)}\right) d x \\
= & -\frac{F_{2}\left(a, x_{2}\right) F_{2}\left(a, x_{3}\right)}{\left(a-x_{1}\right) M(a)(a-b)} \\
& -\frac{F_{2}\left(b, x_{2}\right) F_{2}\left(b, x_{3}\right)}{\left(b-x_{1}\right) M(b)(b-a)} \\
= & \frac{b-a}{\left(a-x_{1}\right)\left(a-x_{2}\right)\left(a-x_{3}\right) M(a)} \\
& -\frac{b-a}{\left(b-x_{1}\right)\left(b-x_{2}\right)\left(b-x_{3}\right) M(b)}
\end{aligned}
$$

i.e.

$$
W_{3}\left(x_{1}, x_{2}, x_{3}\right)=(b-a) \frac{\frac{1}{\left(a-x_{1}\right)\left(a-x_{2}\right)\left(a-x_{3}\right) M(a)}-\frac{1}{\left(b-x_{1}\right)\left(b-x_{2}\right)\left(b-x_{3}\right) M(b)}}{8 \sqrt{\sigma\left(x_{1}\right)} \sqrt{\sigma\left(x_{2}\right)} \sqrt{\sigma\left(x_{3}\right)}}
$$

which is the usual of [1].

$$
\begin{aligned}
\sqrt{\sigma\left(x_{1}\right)} W^{(1)}\left(x_{1}\right)= & \operatorname{Res}_{a, b} W_{2}(x, x) \frac{d x}{2\left(x_{1}-x\right) M(x)} \\
= & \frac{(b-a)^{2}}{32} \operatorname{Res}_{a, b} \frac{d x}{\left(x_{1}-x\right) M(x) \sigma(x)^{2}} \\
= & \frac{(b-a)^{2}}{32} \operatorname{Res}_{a} \frac{d x}{(x-a)^{2}} \frac{1}{\left(x_{1}-x\right) M(x)(x-b)^{2}} \\
& +\frac{(b-a)^{2}}{32} \operatorname{Res}_{b} \frac{d x}{(x-b)^{2}} \frac{1}{\left(x_{1}-x\right) M(x)(x-a)^{2}} \\
= & \left.\frac{(b-a)^{2}}{32}\left(\frac{1}{\left(x_{1}-x\right) M(x)(x-b)^{2}}\right)^{\prime}\right|_{x=a}
\end{aligned}
$$




$$
\begin{array}{ll} 
& +\left.\frac{(b-a)^{2}}{32}\left(\frac{1}{\left(x_{1}-x\right) M(x)(x-a)^{2}}\right)^{\prime}\right|_{x=b} \\
=\quad & \frac{(b-a)^{2}}{32}\left(\frac{1}{M(a)} \frac{-2 x_{1}-b+3 a}{(a-b)^{3}\left(x_{1}-a\right)^{2}}-\frac{M^{\prime}(a)}{M(a)^{2}} \frac{1}{(a-b)^{2}\left(x_{1}-a\right)}\right) \\
& +\frac{(b-a)^{2}}{32}\left(\frac{1}{M(b)} \frac{-2 x_{1}-a+3 b}{(b-a)^{3}\left(x_{1}-b\right)^{2}}-\frac{M^{\prime}(b)}{M(b)^{2}} \frac{1}{(b-a)^{2}\left(x_{1}-b\right)}\right)
\end{array}
$$

which again agrees with [3] and other results in the literature.

\section{Conclusions and prospects}

In this article, we have found a $\phi^{3}$ Feynmann graph formulation for computing all correlations functions to all powers of $N$ in the one-hermitian matrix model. First, it would be interesting to find out to which field theory it corresponds. One is tempted to compare with Liouville's theory (which is not cubic) or to a fermionic theory.

We claim that this approach is more efficient for actual calculations, than the method existing previously in the literature [2, 3]. Indeed, in [2, 3], one has to construct the correlation functions recursively, by expanding them on basis functions which are themselves constructed recursively by taking derivatives with respect to the potential. For instance, one does not get any simplification in the method of [2, 3] by assuming an even potential, or by assuming a quadratic potential. The method presented here, works for fixed potential (for instance quadratic), and does not need to construct any basis of functions.

Another important point for the method presented here, is that it is expressed in terms of geometrical fundamental objects on the spectral curve. This is another evidence of the deep link between tau functions and complex geometry.

There are other expressions in the literature involving Residues of geometrical objects (for instance [33, 29, 4, 5, 13, 15, 28, 26]), namely, only the Bergmann kernel and not the abelian differential. However, we claim that it should be simpler to compute the residue of a function with a simple pole (the abelian differential), than the residue of a function with a double pole (Bergmann kernel).

Moreover, the whole procedure described here, can be applied with very small adaptations to other matrix models, in particular the 2-matrix model, and to nonhermitian matrix models (in particular $\beta=1,2,4$ models), this work is in progress and will be available shortly [20]. In the 2-matrix model with potentials of degree $d_{1}+1$ and $d_{2}+1$, the computation of correlation functions of the first matrix only involves $d_{2}$ vertices (i.e. cubic, quartic, $\ldots, d_{2}+2$-legs-vertex), instead of only one cubic vertex equal to $1 / 2 y(x)$ for the 1-matrix model. This will be further explained in [20]. 
The observable we have not computed in this article is the free energy:

$$
\bar{G}_{0}:=-\frac{1}{N^{2}} \ln Z:=\sum_{h=0}^{\infty} N^{-2 h} G_{0}^{(h)}
$$

The free energy does not appear in the loop equations. It satisfies:

$$
\frac{\partial G_{0}^{(h)}}{\partial V\left(x_{1}\right)}=-W_{1}^{(h)}\left(x_{1}\right)
$$

therefore, in order to compute the free energy, one has to integrate with respect to the potential, i.e. one can no longer keep the potential constant. One would reasonably make the following conjecture for $h \geq 2$ :

$$
G_{0}^{(h)} \rightarrow \sum_{T \in \mathcal{T}_{0}^{(h)}} \mathcal{W}(T)
$$

for example for $h=2$

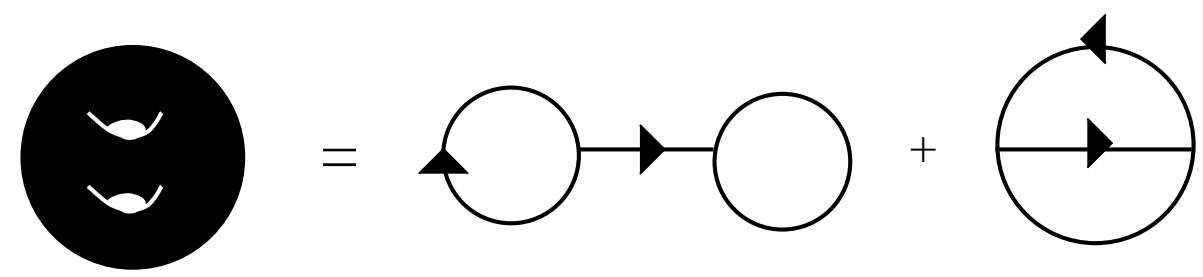

unfortunately, these Feynmann graph don't make sense (the abelian differential diverges at coinciding points). The conjecture is that the $G_{0}^{(h)}$ are related to traces of powers of the laplacian on the spectral curve. For instance it is known that $G_{0}^{(1)}$ is related to the determinant of the laplacian [15].

Aknowledgements: The author wants to thank the EU network EC IHP network (HPRN-CT-1999-000161), as well as the CRM in Montreal where a large part of this research was conducted. The author wants to thank M. Bertola, P. Di Francesco, E. Guitter, J. Harnad, I. Kostov, P. Wiegman, and A. Zabrodin for helpfull and stimulating discussions.

\section{Appendix A Cardinal of $\mathcal{T}_{k}^{(h)}$}

The cardinal of $\mathcal{T}_{k}$ and of $\mathcal{T}_{k}^{(h)}$ can be computed by setting $\mathcal{W}=1$ in 4.40 and in 5.14, and then using the recursion relations 4.35 and 5.9.

One thus gets for $k \geq 3$ :

$$
N_{2}=1 \quad, \quad N_{k}=\sum_{j=1}^{k-2}\left(\begin{array}{c}
k-1 \\
j
\end{array}\right) N_{j+1} N_{k-j}
$$


writing:

$$
r_{0}:=0 \quad, \quad r_{1}:=1 \quad, \quad r_{k}:=\frac{N_{k+1}}{k !}
$$

A.1 becomes for $k \geq 2$ :

$$
r_{k}=\sum_{j=0}^{k} r_{j} r_{k-j}
$$

We introduce the generating function:

$$
R(x):=\sum_{k=0}^{\infty} r_{k} x^{k}
$$

and thus A.3 becomes:

$$
R(x)-x=R^{2}(x)
$$

whose solution is:

$$
R(x)=\frac{1-\sqrt{1-4 x}}{2}=-\frac{1}{2} \sum_{k=1}^{\infty}\left(\begin{array}{l}
\frac{1}{2} \\
k
\end{array}\right)(-4 x)^{k}
$$

which implies:

$$
r_{k}=-\frac{(-4)^{k}}{2}\left(\begin{array}{c}
\frac{1}{2} \\
k
\end{array}\right)=(-1)^{k+1} 2^{2 k-1} \frac{\frac{1}{2}\left(-\frac{1}{2}\right) \ldots\left(\frac{3}{2}-k\right)}{k !}=2^{k-1} \frac{(2 k-3) ! !}{k !}=\frac{2 k-2 !}{k ! k-1 !}
$$

and thus, we obtain 4.37

$$
N_{k}=2^{k-2}(2 k-5) ! !=\frac{2 k-4 !}{k-2 !}
$$

For higher genus, we have for $k \geq 1$ and $h \geq 1$ :

$$
N_{1}^{(0)}:=0 \quad, \quad N_{k}^{(h)}=N_{k+1}^{(h-1)}+\sum_{j=0}^{k-1} \sum_{m=0}^{h}\left(\begin{array}{c}
k-1 \\
j
\end{array}\right) N_{j+1}^{(m)} N_{k-j}^{(h-m)}
$$

writing:

$$
r_{0}^{(0)}:=0 \quad, \quad r_{k}^{(h)}:=\frac{N_{k+1}^{(h)}}{k !}
$$

A.9 becomes for $k \geq 0, h \geq 1$ :

$$
r_{k}^{(h)}=(k+1) r_{k+1}^{(h-1)}+\sum_{j=0}^{k} \sum_{m=0}^{h} r_{j}^{(m)} r_{k-j}^{(h-m)}
$$

We introduce the generating function:

$$
R_{h}(x):=\sum_{k=0}^{\infty} r_{k}^{(h)} x^{k}
$$


and thus A.11 becomes for $h \geq 0$ :

$$
R_{0}(x)=R(x) \quad, \quad R_{h}(x)=R_{h-1}^{\prime}(x)+\sum_{m=0}^{h} R_{m}(x) R_{h-m}(x)
$$

which can also be written for $h \geq 1$ :

$$
(1-2 R(x)) R_{h}(x)=R_{h-1}^{\prime}(x)+\sum_{m=1}^{h-1} R_{m}(x) R_{h-m}(x)
$$

using A.6, it is easy to see, by induction on $h$ that for $h \geq 1$ one has:

$$
R_{h}(x)=s_{h}(1-4 x)^{-\frac{3 h-1}{2}}
$$

where the coefficients $s_{h}$ obey for $h \geq 1$ :

$$
s_{1}=1 \quad, \quad s_{h}=2(3 h-4) s_{h-1}+\sum_{m=1}^{h-1} s_{m} s_{h-m}
$$

or, if we define $s_{0}:=-\frac{1}{2}$, it can be written for any $h \geq 1$ :

$$
0=2(3 h-4) s_{h-1}+\sum_{m=0}^{h} s_{m} s_{h-m}
$$

we introduce the generating function:

$$
S(x):=\sum_{h=0}^{\infty} s_{h} x^{h}
$$

it obeys:

$$
0=S^{2}(x)-\frac{1}{4}+6 x^{2} S^{\prime}(x)-2 x S(x)
$$

If one writes

$$
\xi=\frac{x^{-2 / 3}}{4}
$$

and

$$
S(x)=-x^{\frac{1}{3}} h(\xi)
$$

one has:

$$
\xi=h^{2}(\xi)+h^{\prime}(\xi)
$$

whose solution is;

$$
h(\xi)=\frac{A i^{\prime}(\xi)}{A i(\xi)}=\frac{\int t d t \mathrm{e}^{-\frac{t^{3}}{3}+t \xi}}{\int d t \mathrm{e}^{-\frac{t^{3}}{3}+t \xi}}=\sqrt{\xi} \frac{\int t d t \mathrm{e}^{\xi^{3 / 2}\left(-\frac{t^{3}}{3}+t\right)}}{\int d t \mathrm{e}^{\xi^{3 / 2}\left(-\frac{t^{3}}{3}+t\right)}}=\frac{x^{-1 / 3}}{2} \frac{\int t d t \mathrm{e}^{\frac{1}{8 x}\left(-\frac{t^{3}}{3}+t\right)}}{\int d t \mathrm{e}^{\frac{1}{8 x}\left(-\frac{t^{3}}{3}+t\right)}}
$$


and thus:

$$
S(x)=-\frac{1}{2} \frac{\int t d t \mathrm{e}^{\frac{1}{8 x}\left(-\frac{t^{3}}{3}+t\right)}}{\int d t \mathrm{e}^{\frac{1}{8 x}\left(-\frac{t^{3}}{3}+t\right)}}=-\frac{1}{2}\left(1+\frac{\int t d t \mathrm{e}^{\frac{1}{8 x}\left(-t^{2}-\frac{t^{3}}{3}\right)}}{\int d t \mathrm{e}^{\frac{1}{8 x}\left(-t^{2}-\frac{t^{3}}{3}\right)}}\right)
$$

or

$$
\begin{gathered}
S(x)=-\frac{1}{2}-\sqrt{x} \frac{\int t d t \mathrm{e}^{-\frac{t^{2}}{2}} \mathrm{e}^{-\frac{\sqrt{x} t^{3}}{3}}}{\int d t \mathrm{e}^{-\frac{t^{2}}{2}} \mathrm{e}^{-\frac{\sqrt{x} t^{3}}{3}}} \\
S(x)=-\frac{1}{2}+\frac{\sum_{h=0}^{\infty} \frac{x^{h+1}}{\sum^{2 h+1}(2 h+1) !} \int d t t^{2(3 h+2)} \mathrm{e}^{-t^{2} / 2}}{\sum_{h=0}^{\infty} \frac{x^{h}}{3^{2 h}(2 h) !} \int d t t^{2(3 h)} \mathrm{e}^{-t^{2} / 2}} \\
S(x)=-\frac{1}{2}+\frac{\sum_{h=0}^{\infty} \frac{x^{h+1}(6 h+3) ! !}{3^{2 h+1}(2 h+1) !}}{1+\sum_{h=1}^{\infty} \frac{x^{h}(6 h-1) ! !}{3^{2 h}(2 h) !}}=-\frac{1}{2}+x \frac{\sum_{h=0}^{\infty} \frac{x^{h}(6 h+4) !}{3^{2 h+1} 2^{3 h+2}(2 h+1) !(3 h+2) !}}{\sum_{h=0}^{\infty} \frac{x^{h}(6 h) !}{3^{2 h} 2^{3 h}(2 h) !(3 h) !}}
\end{gathered}
$$

In the end we have:

$$
N_{k}^{(h)}:=\operatorname{Card} \mathcal{T}_{k}^{(h)}=s_{h}(k-1) ! 4^{k-1}\left(\begin{array}{c}
\frac{3(h-1)}{2}+k-1 \\
k-1
\end{array}\right)
$$

The whole function is thus:

$$
\begin{aligned}
R(x, z) & :=\sum_{k} \sum_{h} N_{k}^{(h)} x^{k} z^{h}=\sum_{h} R_{h}(x) z^{h} \\
& =-z \frac{\int d t t \mathrm{e}^{-\frac{z^{2}}{3} t^{3}} \mathrm{e}^{\frac{t}{4}(1-4 x)}}{\int d t \mathrm{e}^{-\frac{z^{2}}{3} t^{3}} \mathrm{e}^{\frac{t}{4}(1-4 x)}}
\end{aligned}
$$

\section{References}

[1] J. Ambjorn, G. Akemann, "New universal spectral correlators" J.Phys. A29 (1996) L555-L560, cond-mat/9606129

[2] G. Akemann, "Universal correlators for multi-arc complex matrix models", Nucl.Phys. B507 (1997) 475-500, hep-th/9702005.

[3] J. Ambjorn, L. Chekhov, C.F. Kristjansen, Yu. Makeenko, "Matrix Model Calculations beyond the Spherical Limit", Nucl.Phys. B404 (1993) 127-172. Erratumibid. B449 (1995) 681.

[4] M. Bertola, "Free Energy of the Two-Matrix Model/dToda Tau-Function", preprint CRM-2921 (2003), hep-th/0306184.

[5] M. Bertola, B. Eynard, "Higher Order Observables of the Two-Matrix Model with semiclassical potentials", in preparation. 
[6] P.M. Bleher and A.R. Its, eds., "Random Matrix Models and Their Applications", MSRI Research Publications 40, Cambridge Univ. Press, (Cambridge, 2001).

[7] G. Bonnet, F. David, B. Eynard, "Breakdown of universality in multi-cut matrix models", J.Phys. A 33 6739-6768 (2000).

[8] E. Brezin, C. Itzykson, G. Parisi, and J. Zuber, Comm. Math. Phys. 59, 35 (1978).

[9] F. David, "Planar diagrams, two-dimensional lattice gravity and surface models", Nucl. Phys. B 257 [FS14] 45 (1985).

[10] P. Deift, T. Kriecherbauer, K. T. R. McLaughlin, S. Venakides, Z. Zhou, "Uniform asymptotics for polynomials orthogonal with respect to varying exponential weights and applications to universality questions in random matrix theory", Commun. Pure Appl. Math. 52, 1335-1425 (1999)

[11] P. Di Francesco, P. Ginsparg, J. Zinn-Justin, "2D Gravity and Random Matrices", Phys. Rep. 254, 1 (1995).

[12] R. Dijkgraaf, C. Vafa, "A Perturbative Window into Non-Perturbative Physics", hep-th/0208048, "On Geometry and Matrix Models", Nucl.Phys. B644 (2002) 21-39, hep-th/0207106, "Matrix Models, Topological Strings, and Supersymmetric Gauge Theories", Nucl.Phys. B644 (2002) 3-20, hep-th/0206255.

[13] B. Dubrovin, Y. Zhang, "Bihamiltonian Hierarchies in 2D Topological Field Theory At One-Loop Approximation", Commun.Math.Phys. 198 (1998) 311-361, hepth/9712232.

[14] F. J. Dyson, "Correlations between the eigenvalues of a random matrix", Comm. Math. Phys 19 (1970) 235-50.

[15] B. Eynard, D. Korotkin, A. Kokotov, " $1 / N^{2}$ correction to free energy in hermitian two-matrix model", http://arXiv.org/abs/hep-th/0401166.

[16] B. Eynard, "Large N expansion of the 2-matrix model", JHEP 01 (2003) 051, hep-th/0210047.

[17] B. Eynard, "Large N expansion of the 2-matrix model, multi-cut case", preprint SPHT03/106, ccsd-00000521, math-ph/0307052.

[18] B. Eynard, "Eigenvalue distribution of large random matrices, from one matrix to several coupled matrices" Nucl. Phys. B506, 633 (1997), cond-mat/9707005. 
[19] B. Eynard, "Correlation functions of eigenvalues of multi-matrix models, and the limit of a time dependent matrix", J. Phys. A: Math. Gen. 31, 8081 (1998), condmat/9801075.

[20] B. Eynard, "Higher correlation functions of the 2-non-hermitian matrix model, to all genus", in preparation.

[21] H.M. Farkas, I. Kra, "Riemann surfaces" 2nd edition, Springer Verlag, 1992.

[22] J.D. Fay, "Theta functions on Riemann surfaces", Springer Verlag, 1973.

[23] P. Ginsparg, Matrix models of 2D gravity (Trieste Summer School, July 1991).

[24] Two dimensional quantum gravity and random surfaces, edited by D. Gross, T. Piran, and S. Weinberg (Jerusalem winter school, World Scientific, 1991).

[25] T. Guhr, A. Mueller-Groeling, H.A. Weidenmuller, "Random matrix theories in quantum physics: Common concepts", Phys. Rep. 299, 189 (1998).

[26] V.A. Kazakov, A. Marshakov, "Complex Curve of the Two Matrix Model and its Tau-function", J.Phys. A36 (2003) 3107-3136, hep-th/0211236.

[27] V.G. Knizhnik, A.M. Polyakov, A.B. Zamolodchikov, Mod. Phys. Lett. A3 (1988) 819.

[28] I. K. Kostov, "Boundary Correlators in 2D Quantum Gravity: Liouville versus Discrete Approach", Nucl.Phys. B658 (2003) 397-416, hep-th/0212194.

[29] I. Krichever, "The $\tau$-Function of the Universal Whitham Hierarchy, Matrix Models and Topological Field Theories", Comm. Pure Appl. Math. 47 (1994), no. 4, 437475 .

[30] M.L. Mehta, Random Matrices,2nd edition, (Academic Press, New York, 1991).

[31] M. Staudacher, "Combinatorial solution of the 2-matrix model", Phys. Lett. B305 (1993) 332-338.

[32] J.J.M. Verbaarshot, "Random matrix model approach to chiral symmetry", Nucl. Phys. Proc. Suppl. 53, 88 (1997).

[33] P. Wiegmann, A. Zabrodin, "Large $\mathrm{N}$ expansion for normal and complex matrix ensembles", hep-th/0309253. 